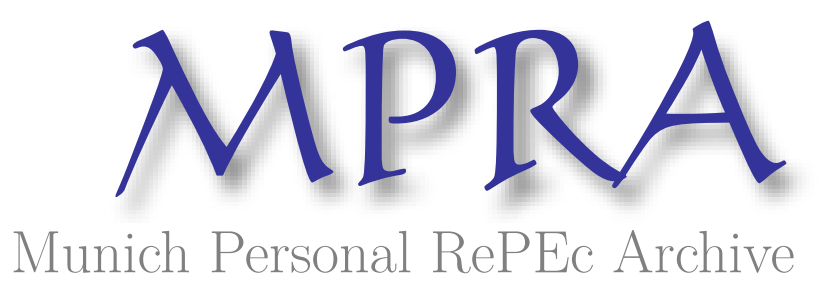

\title{
Time-varying parameters Realized GARCH models for tracking attenuation bias in volatility dynamics
}

Gerlach, Richard and Naimoli, Antonio and Storti, Giuseppe

University of Sydney, University of Salerno, University of Salerno

12 March 2020

Online at https://mpra.ub.uni-muenchen.de/99398/

MPRA Paper No. 99398, posted 07 Apr 2020 14:04 UTC 


\title{
Time-varying parameters Realized GARCH models for tracking attenuation bias in volatility dynamics
}

\author{
1st April 2020
}

\begin{abstract}
This paper proposes novel approaches to the modeling of attenuation bias effects in volatility forecasting. Our strategy relies on suitable generalizations of the Realized GARCH model by Hansen et al. (2012) where the impact of lagged realized measures on the current conditional variance is weighted according to the accuracy of the measure itself at that specific time point. This feature allows assigning more weight to lagged volatilities when they are more accurately measured. The ability of the proposed models to generate accurate forecasts of volatility and related tail risk measures, Value-at-Risk and Expected Shortfall, is assessed by means of an application to a set of major stock market indices. The results of the empirical analysis show that the proposed specifications are able to outperform standard Realized GARCH models in terms of out-of-sample forecast performance under both statistical and economic criteria.
\end{abstract}

JEL Codes: C58, C22,C53.

Keywords: Realized GARCH, Realized Volatility, Realized Quarticity, Attenuation Bias, Measurement Error, Tail Risk Forecasting.

\section{Introduction}

It is widely acknowledged that the use of realized volatility measures (Hansen and Lunde, 2011) can be beneficial for improving the accuracy of volatility forecasts on a daily scale. This is typically done by choosing one of the following approaches.

First, dynamic models can be directly fitted to time series of realized measures. Examples include the Heterogeneous AutoRegressive (HAR) (Corsi, 2009) and the 
class of Multiplicative Error Models (MEM) (Engle, 2002; Engle and Gallo, 2006). A drawback of this approach is that the focus is on the estimation of the expected level of the realized measure, rather than on the estimation of the conditional variance of returns. As clarified in the next section, realized measures are designed to consistently estimate the integrated variance, which is related to but different from, the conditional variance. Namely, in the absence of microstructure noise and jumps, the integrated variance can be interpreted as an unbiased estimator of the conditional variance of returns.

The second approach makes use of time series models for daily returns, e.g. GARCH-type models, where the conditional variance is driven by one or more realized measures. The main idea is to replace a noisy volatility proxy, such as the squared daily returns used in standard GARCH models, with a more efficient realized measure. Differently from the above-mentioned approach, in this case both low (daily returns) and high (realized measures) frequency information is employed in the model. Examples of models in this class include the HEAVY model of Shephard and Sheppard (2010) and the Realized GARCH model of Hansen et al. (2012). These two models are closely related but, nevertheless, are characterized by some distinctive features. Realized GARCH models include a measurement equation, allowing one to gain, in a fully data-driven fashion, deeper insight on the statistical properties of the realized measure and its relationship with the latent volatility. In addition, the measurement equation offers a convenient framework for simulation and generation of multi-step ahead forecasts. Differently, in HEAVY models, the generation of multi-step ahead forecasts is guaranteed by the inclusion of an additional dynamic updating equation for the conditional expectation of the chosen realized measure.

A complication arising with both approaches is that realized measures are noisy estimates of the underlying integrated variance, generating a classical errors-invariables problem. This typically leads to the rise of what is often called attenuation bias. More precisely, the estimated response of the conditional variance to the past realized measure will be biased towards 0, compared to what we would have found replacing the realized measure by the latent integrated variance. The size of this effect is not constant but time-varying since it is directly related to the variability of the volatility measurement error.

Although it is evident that accounting for this time-varying attenuation bias can potentially lead to improved volatility forecasts, this issue has not yet received much attention in the literature. Recently, Bollerslev et al. (2016) found that, in a HAR model, letting the volatility persistence depend on the estimated degree of measurement error leads to some improvement in the model's predictive performance. In the same spirit, Buccheri and Corsi (2019) proposed time-varying parameter HAR models, that can account for both measurement errors and non-linearities in the dynamics of realized measures. Moving to a GARCH framework, Shephard and Xiu (2016) found evidence that, in a GARCH-X model, the magnitude of the response coefficients associated with different realized volatility measures is related to the quality of the measure itself. Finally, Hansen and Huang (2016) observed that the response of the current conditional variance to past unexpected volatility shocks is negatively correlated with the accuracy of the associated realized volatility measure.

Our contribution to the research in this field is threefold. First, we provide 
theoretical insight on the impact of attenuation bias in the Realized GARCH framework. Our theoretical findings are then confirmed by a Monte Carlo simulation study taking a contaminated log-linear Realized GARCH model of order $(1,1)$ as the Data Generating Process. Summarizing the theoretical and empirical evidence, we find that measurement errors in the computation of realized volatility measures lead to: i) downward bias in the volatility reaction parameter, the so called "ARCH" coefficient; and ii) upward bias in the volatility inertial decay parameter, the so called "GARCH" coefficient. These two biases compensate for each other, so that the overall volatility persistence is unaffected. Second, we develop extensions of the standard log-linear Realized GARCH model, that account for time-varying attenuation bias effects in the conditional variance dynamics. This is achieved by allowing the coefficients, of the dynamic volatility updating equation of the log-linear Realized GARCH model, to vary over time as a function of an estimator of the asymptotic variance of the realized measure. For the realized variance estimator, this is given by a rescaling of the integrated quarticity of intra-daily returns, while, for the log-transformed realized variance, Corsi et al. (2008) show that the asymptotic variance depends on the ratio of the integrated quarticity of intra-daily returns to the squared integrated variance. As a consequence, the resulting model will give more weight to lagged volatilities when these are more accurately measured. Third, we empirically assess the impact of accounting for time-varying attenuation bias on the accuracy of volatility and tail-risk forecasts. Our empirical findings on a set of international stock market indices provide evidence that the proposed modeling approach is able to outperform the benchmark Realized GARCH model, both in terms of purely statistical, and also economically meaningful, loss functions. Specifically, the forecasting ability of a given model is assessed under three different criteria. First, the predictive partial log-likelihood (as defined in Hansen et al., 2012) is used to assess the model's ability to predict the conditional distribution of future returns. Second, the QLIKE loss (Patton et al., 2009) is used to rank models according their ability to forecast volatility. Finally, we focus on the ability to accurately predict Value-at-Risk (VaR) and Expected Shortfall (ES) at different confidence levels. Namely, in order to assess the models' performances in forecasting VaR, we rely on the Quantile Loss function (Koenker, 2005), while for evaluating joint forecasts of the pair (VaR, ES) we refer to the class of strictly consistent loss functions proposed by Fissler and Ziegel (2016).

The paper is organized as follows. Section 2 reviews the basic theoretical framework behind the computation of realized measures and Section 3 discusses the Realized GARCH model of Hansen et al. (2012). In Section 4, we then provide theoretical and empirical insight on the occurrence of attenuation bias effects in Realized GARCH models while Section 5 illustrates the proposed time-varying parameters Realized GARCH models. Section 6 focuses on the associated estimation and inference procedures and Section 7 presents the results of the empirical application. Finally, Section 8 concludes. 


\section{Realized measures: a short review}

In recent years, the availability of high-frequency financial market data has enabled researchers to build reliable measures of the latent daily volatility, based on the use of intra-daily returns. In the econometric and financial literature, these are widely known as realized volatility measures. The theoretical background to these measures is given by the dynamic specification of the price process in continuous time. Formally, let the logarithmic price $p_{t}$ of a financial asset be determined by the stochastic differential process

$$
d p_{t}=\mu_{t} d t+\sigma_{t} d W_{t} \quad 0 \leq t \leq T,
$$

where $\mu_{t}$ and $\sigma_{t}$ are the drift and instantaneous volatility processes, respectively, whilst $W_{t}$ is a standard Brownian motion; $\sigma_{t}$ is assumed to be independent of $W_{t}$. Under assumption of a frictionless market, the logarithmic price $p_{t}$ follows a semi-martingale process.

In that case, given a sequence of partitions $t-1=\tau_{0} \leq \tau_{1} \leq \ldots \leq \tau_{M}=t$, the Quadratic Variation $(Q V)$ of log-returns $r_{t}=p_{t}-p_{t-1}$, given by

$$
Q V_{t}=\operatorname{plim}_{M \rightarrow \infty} \sum_{j=0}^{M-1}\left(p_{\tau_{j+1}}-p_{\tau_{j}}\right)^{2}
$$

coincides with the Integrated Variance $(I V)$

$$
I V_{t}=\int_{t-1}^{t} \sigma_{s}^{2} d s
$$

In the absence of microstructure noise and measurement errors, Barndorff-Nielsen and Shephard (2002) show that $I V$ is consistently estimated by Realized Volatility $(R V)$

$$
R V_{t}=\sum_{i=1}^{M} r_{t, i}^{2}
$$

where

$$
r_{t, i}=p_{t-1+i \Delta}-p_{t-1+(i-1) \Delta}
$$

is the $i$-th $\Delta$-period intraday return, $M=1 / \Delta$. Although $I V$ and the conditional variance of returns do not coincide, there is a precise relationship between these two quantities: under standard integrability conditions (Andersen et al., 2001) it can be shown that

$$
E\left(I V_{t} \mid \mathscr{F}_{t-1}\right)=\operatorname{var}\left(r_{t} \mid \mathscr{F}_{t-1}\right)
$$

where $\mathscr{F}_{t-1}$ denotes the information set at time $(t-1)$. In other words, the optimal forecast of $I V$ can be interpreted as the conditional variance of returns and the difference between these two quantities is given by a zero mean error.

Barndorff-Nielsen and Shephard (2002) show that $R V$ consistently estimates the true latent volatility, when $\Delta \longrightarrow 0$. They also find that, conditional on the observed realization of $I V_{t}$, the asymptotic distribution of $R V_{t}$ is Gaussian

$$
\frac{\sqrt{M}\left(R V_{t}-I V_{t}\right)}{\sqrt{2 I Q_{t}}} \rightarrow N(0,1),
$$


where $I Q_{t}=\int_{t-1}^{t} \sigma_{s}^{4} d s$ is the Integrated Quarticity $(I Q)$. This, in turn, can be consistently estimated as

$$
R Q_{t}=\frac{M}{3} \sum_{i=1}^{M} r_{t, i}^{4}
$$

Replacing $I Q_{t}$ by $R Q_{t}$ in equation (5) still gives

$$
\frac{\sqrt{M}\left(R V_{t}-I V_{t}\right)}{\sqrt{2 R Q_{t}}} \rightarrow N(0,1) .
$$

In financial modeling, the use of $\log \left(R V_{t}\right)$ is often preferred to the "plain" $R V_{t}$ estimator, due to its favourable finite sample properties (see Corsi et al. (2008), among others). The approximate asymptotic distribution of $\log \left(R V_{t}\right)$ can be shown to be

$$
\frac{\left(\log \left(R V_{t}\right)-\log \left(I V_{t}\right)\right)}{\sqrt{\frac{2 R Q_{t}}{M R V_{t}^{2}}}} \rightarrow N(0,1) .
$$

Furthermore, Corsi et al. (2008) provide empirical evidence that, in a HAR model, choosing the logarithmic realized variance as a dependent variable and allowing for time-varying volatility of realized volatility leads to substantial improvements in fit and forecasting performance.

\section{Realized GARCH models}

The Realized GARCH (RGARCH) model, introduced by Hansen et al. (2012), extends the class of GARCH models by first replacing squared returns, as the driver of the volatility dynamics, with a more efficient proxy, such as a $R V$ measure. With this change alone, the resulting specification can be seen as a GARCH-X model, where the realized measure is used as an explanatory variable. A second extension is that the Realized GARCH "completes" the GARCH-X by adding a measurement equation, explicitly modeling the contemporaneous relationship between the realized measure and the latent conditional variance.

Formally, let $\left\{r_{t}\right\}$ be a time series of financial returns and $\left\{x_{t}\right\}$ be a time series of realized measures of volatility. Focus here is on the logarithmic RGARCH model, defined via

$$
\begin{aligned}
r_{t} & =\mu_{t}+\sqrt{h_{t}} z_{t} \\
\widetilde{h}_{t} & =\omega+\beta \widetilde{h}_{t-1}+\gamma \widetilde{x}_{t-1}, \\
\widetilde{x}_{t} & =\xi+\varphi \widetilde{h}_{t}+\tau\left(z_{t}\right)+u_{t},
\end{aligned}
$$

where $\widetilde{x}_{t}=\log \left(x_{t}\right), h_{t}=\operatorname{var}\left(r_{t} \mid \mathscr{F}_{t-1}\right)$ is the conditional variance and $\widetilde{h}_{t}=\log \left(h_{t}\right)$. To simplify the exposition, in the remainder, it is assumed that $\mu_{t}=E\left(r_{t} \mid \mathscr{F}_{t-1}\right)=0$. The innovations $z_{t}$ and $u_{t}$ are assumed to be mutually independent, with $z_{t} \underset{\text { iid }}{\sim}(0,1)$ and $u_{t} \underset{i i d}{\sim}\left(0, \sigma_{u}^{2}\right)$. 
The function $\tau\left(z_{t}\right)$ can accommodate leverage effects, since it captures the dependence between returns and future volatility. A common choice (see e.g. Hansen et al. (2012)), found to be empirically satisfactory, is

$$
\tau\left(z_{t}\right)=\tau_{1} z_{t}+\tau_{2}\left(z_{t}^{2}-1\right) .
$$

Substituting the measurement equation into the volatility equation, the model implies an $\mathrm{AR}(1)$ representation for $\widetilde{h}_{t}$

$$
\widetilde{h}_{t}=(\omega+\xi \gamma)+(\beta+\varphi \gamma) \widetilde{h}_{t-1}+\gamma w_{t-1},
$$

where $w_{t}=\tau\left(z_{t}\right)+u_{t}$ and $E\left(w_{t}\right)=0$. The coefficient $(\beta+\varphi \gamma)$ reflects the persistence in (the logarithm of) volatility, whereas $\gamma$ represents the impact of both the lagged return and realized measure on future (log-)volatility. To ensure that the $\widetilde{h}_{t}$ is stationary, the required restriction is $\beta+\varphi \gamma<1$. Estimation of model parameters can be easily performed by numerically maximizing a Gaussian Quasi-Likelihood function. Regarding the statistical properties of these estimates, Li et al. (2019) have recently formally proved their consistency and asymptotic normality for the log-linear RGARCH model.

Compared to the linear RGARCH, the log-linear specification has two main advantages: first, it is more flexible, since no constraints on the parameters are required in order to ensure positivity of the conditional variance, which holds automatically by construction; second, the logarithmic transformation substantially reduces the heteroskedasticity of the measurement equation error term. For these reasons, this paper exclusively focuses on the log-linear specification of the Realized GARCH model.

\section{Attenuation-bias effects in RGARCH models}

In this section, our aim is to provide some insight on how attenuation bias effects can arise in RGARCH models. In order to simplify the exposition, without implying any loss of generality, we assume that there are no leverage effects in the measurement equation $\left(\tau_{1}=\tau_{2}=0\right)$ and exclude complications related to microstructure noise and jumps. Also, in the remainder of this section we assume stationarity of the RGARCH processes considered.

To start, let us consider a simple log-linear RGARCH model of order $(1,0)$, where the realized measure is replaced by the latent $I V_{t}$. Referring to the notation defined in the previous section, the resulting specification can be reformulated as an $\operatorname{AR}(1)$ for $\widetilde{I V}_{t}=\log \left(I V_{t}\right)$

$$
\widetilde{I V}_{t}=\mu_{I}+\pi_{I} \widetilde{I V}_{t-1}+u_{t, I}
$$

where $u_{t, I}$ is assumed to be a sequence of zero mean iid errors with finite variance $\sigma_{u, I}^{2}$, $\mu_{I}=\omega_{I} \varphi_{I}+\xi_{I}$ and $\pi_{I}=\varphi_{I} \gamma_{I}$, with the subscript $I$ indicating that the parameters refer to the model fitted using the true $I V_{t}$. In addition, we assume $\gamma_{I}>0$ and $\beta_{I} \geq 0$, consistent with recurrent empirical evidence on the dynamics of time series of realized variances. 
The integrated variance is a latent variable and, in real data application, it can be approximated by the realized variance. Letting $\varepsilon_{t}$ be a series of iid measurement errors with finite variance $\sigma_{\varepsilon}^{2}$, we can write

$$
\widetilde{R V}_{t}=\widetilde{I V}_{t}+\varepsilon_{t},
$$

where $\widetilde{R V}_{t}=\log \left(R V_{t}\right)$. It can be shown, by standard arguments, that equation (13) implies an ARMA $(1,1)$ model for $\widetilde{R V}_{t}$ (see Bollerslev et al. (2016))

$$
\widetilde{R V}_{t}=\mu_{I}+\pi_{I} \widetilde{R V}_{t-1}+\varepsilon_{t}-\pi_{I} \varepsilon_{t-1}+u_{t, I} .
$$

Assume now that a misspecified $\mathrm{AR}(1)$ model is fitted to $\widetilde{R V}_{t}$

$$
\widetilde{R V}_{t}=\omega_{R}+\pi_{R} \widetilde{R V}_{t-1}+u_{t, R},
$$

where $u_{t, R}$ is an iid sequence of zero mean errors with variance $\sigma_{u, R}^{2}$. Letting $c_{R}(k)=$ $\operatorname{cov}\left(\widetilde{R V}_{t}, \widetilde{R V}_{t-k}\right)$ and $c_{I}(k)=\operatorname{cov}\left(\widetilde{I V}_{t}, \widetilde{I V}_{t-k}\right)$, for $k>0$, by the assumptions made on $\varepsilon_{t}, u_{t, I}$ and $u_{t, R}$

$$
c_{R}(1)=c_{I}(1)=\pi_{I} c_{I}(0)
$$

and

$$
c_{R}(0)=c_{I}(0)+\sigma_{\varepsilon}^{2}
$$

It then easily follows that

$$
\pi_{R}=\frac{\pi_{I} c_{I}(0)}{c_{I}(0)+\sigma_{\varepsilon}^{2}}=\pi_{I}\left(1+\frac{\sigma_{\varepsilon}^{2}}{c_{I}(0)}\right)^{-1},
$$

leading to the conclusion, in line with the findings of Bollerslev et al. (2016), that modeling the noisy $R V_{t}$, instead of the latent $I V_{t}$, implies an autoregressive coefficient $\pi_{R}$ lower than that characterizing the dynamics of the latent $I V_{t}$. Equation (17) clearly shows that the impact of this attenuation bias directly depends on the noise variance ratio $\frac{\sigma_{\varepsilon}^{2}}{\operatorname{var}\left(I_{t}\right)}$ : higher ratios correspond to more substantial reductions in volatility persistence.

Since the empirical properties of the observed time series of financial returns usually require working with models of order $(1,1)$, it is of interest to extend our investigation to consider the impact of attenuation bias in this setting. Assuming a $\operatorname{RGARCH}(1,1)$ for $I V_{t}$ implies that $I V_{t}$ follows the ARMA $(1,1)$ model

$$
\widetilde{I V}_{t}=\mu_{I}+\pi_{I} \widetilde{I V}_{t-1}-\beta_{I} u_{t-1, I}+u_{t, I},
$$

where the autoregressive coefficient is now given by $\pi_{I}=\beta_{I}+\varphi_{I} \gamma_{I}$, with $\beta_{I}>0$. By standard theory, the following recursion holds

$$
\rho_{I}(k)=\pi_{I} \rho_{I}(k-1), \quad \forall k>1,
$$

where $\rho_{I}(k)=c_{I}(k) / c_{I}(0)$ is the lag- $k$ autocorrelation function of $\widetilde{I V}_{t}$. This implies that

$$
\pi_{I}=\frac{\rho_{I}(k)}{\rho_{I}(k-1)} .
$$


Also, it can be shown (see (Bollerslev, 1988), among others) that

$$
\rho_{I}(1)=\frac{\left(1-\pi_{I} \beta_{I}\right)\left(\pi_{I}-\beta_{I}\right)}{1+\beta_{I}^{2}-2 \pi_{I} \beta_{I}} .
$$

The value of $\beta_{I}$ can be then obtained as the solution to the following quadratic equation (see Kristensen and Linton (2006))

$$
\beta_{I}^{2}+b_{I} \beta_{I}+1=0,
$$

where

$$
b_{I}=-\frac{\pi_{I}^{2}+1-2 \pi_{I} \rho_{I}(1)}{\pi_{I}-\rho_{I}(1)} .
$$

For $b_{I}<-2$, a well defined solution for $\beta_{I}$ in (21) is given by

$$
\beta_{I}=\frac{-b_{I}-\sqrt{b_{I}^{2}-4}}{2} .
$$

The other available solution is not admissible since it is the reciprocal of the previous one and leads to values $\beta_{I}>1$, in contradiction with the stationarity assumption. Taking the same approach as for the $(1,0)$ case, let us now assume that a misspecified $\operatorname{ARMA}(1,1)$ model is identified for $R V_{t}$

$$
\widetilde{R V}_{t}=\mu_{R}+\pi_{R} \widetilde{R V}_{t-1}-\beta_{R} u_{t-1, R}+u_{t, R} .
$$

By (15) and (19), it then follows that

$$
\pi_{R}=\frac{\rho_{R}(k)}{\rho_{R}(k-1)}=\frac{\rho_{I}(k)}{\rho_{I}(k-1)}=\pi_{I},
$$

so that the two models for $I V_{t}$ and $R V_{t}$ will be characterized by the same AR coefficient. Regarding $\beta_{R}$, as previously shown for the $I V$ model, the value of $\beta_{R}$, for $b_{R}<-2$, can be obtained as

$$
\beta_{R}=\frac{-b_{R}-\sqrt{b_{R}^{2}-4}}{2},
$$

where

$$
b_{R}=-\frac{\pi_{I}^{2}+1-2 \pi_{I} \rho_{R}(1)}{\pi_{I}-\rho_{R}(1)} .
$$

It can be easily shown that $\beta_{R}$ is characterized by an upward bias with respect to $\beta_{I}$ and that this bias tends to increase with the variance of the measurement error $\varepsilon_{t}$. Since $\pi_{I}=\pi_{R}$, the upward bias in $\beta_{R}$ will, in turn, correspond to a downward bias in the value of $\gamma_{R}$. To start, remind that $c_{R}(0)>c_{I}(0)$ so that $\rho_{R}(1)<\rho_{I}(1)$. Differentiating $\beta_{R}$ with respect to $\rho_{R}(1)$ then leads to the following expression

$$
\frac{\partial \beta_{R}}{\partial \rho_{R}(1)}=\frac{\partial \beta_{R}}{\partial b_{R}} \frac{\partial b_{R}}{\partial \rho_{R}(1)}=-\frac{1}{2}\left(1+\frac{b_{R}}{\sqrt{b_{R}^{2}-4}}\right) \frac{\pi_{I}^{2}-1}{\left(\pi_{I}-\rho_{R}(1)\right)^{2}}
$$


for $b_{R}<-2$ and $0<\pi_{I}<1$, where the latter condition follows from the stationarity assumption. Under these constraints, it is easy to show that the derivative in (23) will always be negative for all admissibile $\rho_{R}(1)$ values. So, as $\sigma_{\varepsilon}^{2}$ increases, by (16) $\rho_{R}(1)$ will decrease leading to an increase in the value of $\beta_{R}$.

In order to better illustrate and interpret the relationship between the parameters of RGARCH models of order $(1,1)$ and the measurement error variance, we have performed a Monte Carlo simulation study. The structure of the simulation process can be summarized as follows:

1. Generate $z_{t} \underset{i i d}{\sim}(0,1)$ and $u_{t, I} \underset{i i d}{\sim} N\left(0, \sigma_{u, I}^{2}\right), \quad$ for $t=1, \ldots, T$.

2. Generate an artificial $\log \left(I V_{t}\right)$ series from the $\operatorname{ARMA}(1,1)$ model

$$
\widetilde{I V}_{t}=\mu_{I}+\pi_{I} \widetilde{I V}_{t-1}+w_{t, I}-\beta_{I} w_{t-1, I}, \quad \text { for } t=1, \ldots, T,
$$

where $w_{t, I}=\tau_{I}\left(z_{t}\right)+u_{t, I}$.

3. Generate returns from a $\operatorname{RGARCH}(1,1)$ for $\widetilde{I V}_{t}$.

4. Contaminate $\widetilde{I V}_{t}$ by an additive measurement error $\left(\varepsilon_{t} \underset{\text { iid }}{\sim} N\left(0, \sigma_{\varepsilon}^{2}\right)\right.$ ), in order to generate an artificial $\widetilde{R V}_{t}$ series.

5. Using the ML method, fit a $\operatorname{RGARCH}(1,1)$ model using $\widetilde{R V}_{t}$ as a realized measure.

6. Repeat steps (i)-(v) for $n_{\text {sim }}$ times.

The above design is implemented setting $n_{\text {sim }}=1000$ and $T=2000$, after discarding the first 1000 observations taken as burn-in period. For the distribution of $z_{t}$ two different settings have been considered: $z_{t} \underset{\text { iid }}{\sim} N(0,1)$ and $z_{t} \underset{i i d}{\sim} \sqrt{\frac{v-2}{v}} t(v)$, with $v=5$. Also, in order to illustrate the impact of the noise variance ratio $\left(\sigma_{\varepsilon} / \sigma_{u, I}\right)^{2}$ on the magnitude of the attenuation bias, keeping the value of $\sigma_{u, I}$ fixed to 0.4 , three different values of the measurement error standard deviation $\sigma_{\varepsilon}$ have been considered: $\sigma_{\varepsilon} \in$ $(0.2,0.4,0.6)$. Finally, regarding the coefficients of the dynamic volatility equation, in order to mimic different empirical settings, three different sets of parameter values have been considered. The parameters of the simulated DGPs have been reported in the left panel (columns 1-10) of Table 1. The last nine columns, in the right panel, summarize the simulation results in terms of: simulated mean, relative bias and standard deviation of the estimated $\left(\gamma_{R}, \beta_{R}, \pi_{R}\right)$ coefficients. The simulation confirms our theoretical findings: the $\beta_{R}$ and $\gamma_{R}$ coefficients are affected by upward and downward biases, respectively, while the estimates of $\pi_{R}$ are approximately unbiased. In addition, we find that, as $\sigma_{\varepsilon}$ increases, for both $\beta_{R}$ and $\gamma_{R}$, the estimated bias tends to increase in modulus. For $\beta_{R}$, we also find that, for fixed $\pi_{I}$, the value of the estimated relative bias tends to increase as $\gamma_{I}$ increases and $\beta_{I}$ decreases.

In conclusion of our discussion, some remarks should be made. In the simulation, a simplified setting is considered, in which the realized measurement error is assumed homoskedastic. However, the theoretical results reviewed in Section 2 suggest the 
Table 1: Simulated DGP settings and coefficients (columns 1-10), average estimate, relative bias and Monte Carlo standard error of the estimated parameters $\gamma_{R}, \beta_{R}$ and $\pi_{R}$, for $n_{\text {sim }}=1000$ simulations from $\operatorname{RGARCH}(1,1)$ model (columns 11-19). Key to table: $\bar{\theta}$ : average of estimated $\theta$ values from the $n_{\text {sim }}$ simulated series; $R B\left(\theta_{I}\right)=\frac{\bar{\theta}_{R}-\theta_{I}}{\theta_{I}} \times 100$ (percentage relative bias); $\sigma_{\theta}$ : Monte Carlo standard errors of estimated $\theta$ values from the $n_{\text {sim }}$ simulated series.

\begin{tabular}{|c|c|c|c|c|c|c|c|c|c|c|c|c|c|c|c|c|c|c|}
\hline \multirow{2}{*}{\multicolumn{10}{|c|}{ DGP design }} & \multicolumn{9}{|c|}{ Summary of estimated parameters } \\
\hline & & & & & & & & & & \multicolumn{3}{|c|}{ Average } & \multicolumn{3}{|c|}{ Relative Bias } & \multicolumn{3}{|c|}{ Standard Error } \\
\hline$z_{t}$ & $\omega_{I}$ & $\gamma_{I}$ & $\beta_{I}$ & $\xi_{I}$ & $\varphi_{I}$ & $\tau_{1, I}$ & $\tau_{2, I}$ & $\sigma_{u, I}$ & $\sigma_{\varepsilon}$ & $\overline{\gamma_{R}}$ & $\overline{\beta_{R}}$ & $\overline{\pi_{R}}$ & $R B\left(\gamma_{I}\right)$ & $R B\left(\beta_{I}\right)$ & $R B\left(\pi_{I}\right)$ & $\sigma_{\gamma_{R}}$ & $\sigma_{\beta_{R}}$ & $\sigma_{\pi_{R}}$ \\
\hline$N(0,1)$ & 0.005 & 0.30 & 0.60 & 0.00 & 1.00 & -0.05 & 0.10 & 0.40 & 0.20 & 0.260 & 0.637 & 0.897 & -13.333 & 6.167 & -0.333 & 0.031 & 0.028 & 0.016 \\
\hline$N(0,1)$ & 0.005 & 0.40 & 0.50 & 0.00 & 1.00 & -0.05 & 0.10 & 0.40 & 0.20 & 0.347 & 0.551 & 0.898 & -13.250 & 10.200 & -0.222 & 0.032 & 0.027 & 0.014 \\
\hline$N(0,1)$ & 0.005 & 0.60 & 0.30 & 0.00 & 1.00 & -0.05 & 0.10 & 0.40 & 0.20 & 0.515 & 0.382 & 0.897 & -14.167 & 27.333 & -0.333 & 0.032 & 0.026 & 0.012 \\
\hline$N(0,1)$ & 0.005 & 0.30 & 0.60 & 0.00 & 1.00 & -0.05 & 0.10 & 0.40 & 0.40 & 0.194 & 0.703 & 0.896 & -35.333 & 17.167 & -0.444 & 0.027 & 0.029 & 0.019 \\
\hline$N(0,1)$ & 0.005 & 0.40 & 0.50 & 0.00 & 1.00 & -0.05 & 0.10 & 0.40 & 0.40 & 0.257 & 0.640 & 0.897 & -35.750 & 28.000 & -0.333 & 0.028 & 0.029 & 0.016 \\
\hline$N(0,1)$ & 0.005 & 0.60 & 0.30 & 0.00 & 1.00 & -0.05 & 0.10 & 0.40 & 0.40 & 0.387 & 0.510 & 0.896 & -35.500 & 70.000 & -0.444 & 0.028 & 0.027 & 0.014 \\
\hline$N(0,1)$ & 0.005 & 0.30 & 0.60 & 0.00 & 1.00 & -0.05 & 0.10 & 0.40 & 0.60 & 0.140 & 0.756 & 0.895 & -53.333 & 26.000 & -0.556 & 0.022 & 0.032 & 0.023 \\
\hline$N(0,1)$ & 0.005 & 0.40 & 0.50 & 0.00 & 1.00 & -0.05 & 0.10 & 0.40 & 0.60 & 0.190 & 0.707 & 0.896 & -52.500 & 41.400 & -0.444 & 0.023 & 0.030 & 0.019 \\
\hline$N(0,1)$ & 0.005 & 0.60 & 0.30 & 0.00 & 1.00 & -0.05 & 0.10 & 0.40 & 0.60 & 0.289 & 0.610 & 0.897 & -51.833 & 103.333 & -0.333 & 0.025 & 0.028 & 0.015 \\
\hline$t(0,1,5)$ & 0.005 & 0.30 & 0.60 & 0.00 & 1.00 & -0.05 & 0.10 & 0.40 & 0.20 & 0.267 & 0.629 & 0.897 & -11.000 & 4.833 & -0.333 & 0.032 & 0.027 & 0.015 \\
\hline$t(0,1,5)$ & 0.005 & 0.40 & 0.50 & 0.00 & 1.00 & -0.05 & 0.10 & 0.40 & 0.20 & 0.359 & 0.542 & 0.898 & -10.250 & 8.400 & -0.222 & 0.041 & 0.026 & 0.013 \\
\hline$t(0,1,5)$ & 0.005 & 0.60 & 0.30 & 0.00 & 1.00 & -0.05 & 0.10 & 0.40 & 0.20 & 0.534 & 0.368 & 0.898 & -11.000 & 22.667 & -0.222 & 0.048 & 0.027 & 0.012 \\
\hline$t(0,1,5)$ & 0.005 & 0.30 & 0.60 & 0.00 & 1.00 & -0.05 & 0.10 & 0.40 & 0.40 & 0.203 & 0.693 & 0.897 & -32.333 & 15.500 & -0.333 & 0.031 & 0.030 & 0.017 \\
\hline$t(0,1,5)$ & 0.005 & 0.40 & 0.50 & 0.00 & 1.00 & -0.05 & 0.10 & 0.40 & 0.40 & 0.275 & 0.621 & 0.897 & -31.250 & 24.200 & -0.333 & 0.035 & 0.030 & 0.015 \\
\hline$t(0,1,5)$ & 0.005 & 0.60 & 0.30 & 0.00 & 1.00 & -0.05 & 0.10 & 0.40 & 0.40 & 0.409 & 0.491 & 0.898 & -31.833 & 63.667 & -0.222 & 0.048 & 0.033 & 0.013 \\
\hline$t(0,1,5)$ & 0.005 & 0.30 & 0.60 & 0.00 & 1.00 & -0.05 & 0.10 & 0.40 & 0.60 & 0.154 & 0.739 & 0.893 & -48.667 & 23.167 & -0.778 & 0.034 & 0.063 & 0.062 \\
\hline$t(0,1,5)$ & 0.005 & 0.40 & 0.50 & 0.00 & 1.00 & -0.05 & 0.10 & 0.40 & 0.60 & 0.207 & 0.689 & 0.896 & -48.250 & 37.800 & -0.444 & 0.032 & 0.032 & 0.018 \\
\hline$t(0,1,5)$ & 0.005 & 0.60 & 0.30 & 0.00 & 1.00 & -0.05 & 0.10 & 0.40 & 0.60 & 0.313 & 0.586 & 0.897 & -47.833 & 95.333 & -0.333 & 0.039 & 0.035 & 0.015 \\
\hline
\end{tabular}


assumption of homoskedastic measurement errors is an evident over-simplification. In the presence of a time-varying measurement error variance, the size of the attenuation bias, for both $\gamma$ and $\beta$, is expected to be time-varying as a function of the value of this variance. This issue is addressed in the next section, proposing and discussing extensions of the standard RGARCH model that can account for timevarying attenuation bias effects.

\section{Time-Varying Coefficient Realized GARCH models with dynamic attenuation bias}

In the previous section, evidence is provided on the impact that measurement errors, arising in the approximation of $I V$ via the discretely sampled $R V$, can have on the dynamic properties of the conditional variance in Realized GARCH models. Here, relying on this evidence, we propose a generalization of the basic Realized GARCH specification, accounting for dynamic attenuation bias effects; effects due to the timevarying variability of measurement errors in ex-post volatility estimation. As in Bollerslev et al. (2016), a natural solution to deal with this issue is to consider timevarying parameter models, where the response of $\log \left(h_{t}\right)$ to the lagged realized measure indirectly depends on the value of $\sigma_{u_{R}, t}^{2}$, through the ratio $R Q_{t-1} / R V_{t-1}^{2}$.

Practical implementation of these ideas in the RGARCH framework leads to the Time-Varying Realized GARCH (TV-RGARCH) model, as defined by the following equations

$$
\begin{aligned}
\widetilde{h}_{t} & =\omega+\beta_{t} \widetilde{h}_{t-1}+\gamma_{t} \widetilde{R V}_{t-1}, \\
\widetilde{R V}_{t} & =\xi_{R}+\varphi_{R} \widetilde{h}_{t}+\tau_{R}\left(z_{t}\right)+u_{t, R}, \\
\gamma_{t} & =\gamma+\gamma_{1} \widetilde{Y}_{t-1} \\
\beta_{t} & =\beta+\beta_{1} \widetilde{Y}_{t-1},
\end{aligned}
$$

where $\widetilde{R Q}_{t}=\log \left(\sqrt{R Q_{t}}\right), \widetilde{Y}_{t}=\log \left(Y_{t}\right)=\log \left(\sqrt{R Q_{t}} / R V_{t}\right)=\widetilde{R Q}_{t}-\widetilde{R V}_{t}$. Consistently with the evidence provided in Section 4, the fitted values of the $\gamma_{1}$ and $\beta_{1}$ coefficients are expected to have negative and positive signs, respectively. If this holds, at time $t-1$, lower values of $\widetilde{Y}_{t-1}$ will correspond to higher (lower) values of $\gamma_{t}\left(\beta_{t}\right)$. It can be immediately noted that the RGARCH model is nested in the TV-RGARCH specification for $\gamma_{1}=\beta_{1}=0$.

By simple algebra, the TV-RGARCH model can be further generalized by replacing the specifications in equations (26)-(27) by the following

$$
\begin{aligned}
& \gamma_{t}=\gamma+\gamma_{1} \widetilde{R Q}_{t-1}+\gamma_{2} \widetilde{R V}_{t-1}, \\
& \beta_{t}=\beta+\beta_{1} \widetilde{R Q}_{t-1}+\beta_{2} \widetilde{R V}_{t-1} .
\end{aligned}
$$

We call the resulting model Extended TV-RGARCH (ETV-RGARCH). Equations (28) 
and (29) can further be re-parameterized setting

$$
\begin{aligned}
& \gamma_{2}=-\gamma_{1}+\eta_{\gamma}, \\
& \beta_{2}=-\beta_{1}+\eta_{\beta},
\end{aligned}
$$

making evident that the ETV-RGARCH nests the TV-RGARCH model for $\eta_{\gamma}=\eta_{\beta}=$ 0 . So, under the null of a TV-RGARCH model, we have $\gamma_{2}=-\gamma 1$ and $\beta_{2}=-\beta_{1}$. Thus, the estimated $\gamma_{2}$ and $\beta_{2}$ coefficients are expected to be, respectively, positive and negative.

In order to make the (E)TV-RGARCH model dynamically complete and allow the generation of multi-step ahead forecasts, we need to augment equations (24)-(27) with a further measurement equation for $\widetilde{R Q}_{t}$

$$
\widetilde{R Q}_{t}=\xi_{Q}+\varphi_{Q} \widetilde{h}_{t}+\tau_{Q}\left(z_{t}\right)+u_{t, Q} .
$$

Conditional on $\mathscr{F}_{t-1}$, we assume that $\left(u_{t, R}, u_{t, Q}\right)$ and $z_{t}$ are stochastically independent and

$$
\left(\begin{array}{l}
u_{t, R} \\
u_{t, Q}
\end{array} \mid \mathscr{F}_{t-1}\right) \underset{\text { iid }}{\sim} \operatorname{MVN}\left(\mathbf{0}, \Sigma_{\mathbf{u}}\right), \quad \Sigma_{\mathbf{u}}=\left(\begin{array}{cc}
\sigma_{u, R}^{2} & \rho_{R, Q} \sigma_{u, R} \sigma_{u, Q} \\
\rho_{R, Q} \sigma_{u, R} \sigma_{u, Q} & \sigma_{u, Q}^{2}
\end{array}\right),
$$

where the notation $\operatorname{MVN}_{2}(\mu, \Sigma)$ indicates a bivariate Normal distribution with expectation $\mu$ and variance-covariance matrix $\Sigma$.

Remark 1. We model $u_{t, R}$, the error term in the measurement equation for $\widetilde{R V}_{t}$, as being conditionally homoskedastic. This assumption is indeed not central to our approach and could be easily relaxed. In this respect, recalling the discussion in Section 4, the term $u_{t, R}$, being given by some function of two different error sources, has a complex form. Of these two error sources: the first is given by the random measurement error related to the discrepancy between the log-transformed realized measure and the latent $\widetilde{I V}_{t}$, that is $\varepsilon_{t}$, in the example provided in Section 4; the second source of error is related to the discrepancy between $\widetilde{I V}_{t}$ and the log-transformed conditional variance $\widetilde{h}_{t}$, that is $w_{t, I}=u_{t, I}+\tau_{I}\left(z_{t}\right)$, adding leverage effects to the example provided in Section 4. While it could be a reasonable simplification to assume that $u_{t, I}$ and $z_{t}$ are homoskedastic, recall Section 2, the same of course does not hold for the realized measurement error $\varepsilon_{t}$. So, conditional heteroskedasticity of $\varepsilon_{t}$ could potentially provide support for the hypothesis of conditional heteroskedasticity of $u_{t, R}$. However, the size of this effect, and its empirical detectability, will inevitably depend on the relative variabilities of the components of $u_{t, R}$, i.e. $\varepsilon_{t}$ and $w_{t, I}$. To investigate the presence of conditional heteroskedasticity in the $u_{t, R}$ series, as a robustness check, we have considered an alternative model specification where the conditional variance of the $R V$ measurement equation noise is time-varying, i.e.

$$
\left(u_{t, R} \mid \mathscr{F}_{t-1}\right) \underset{\text { iid }}{\sim} N\left(0, \sigma_{u_{R}, t}^{2}\right) .
$$

Since the variance of the realized measurement error $\varepsilon_{t}$ is a function of the ratio $I Q_{t} / I V_{t}^{2}$, it seems natural to model $\sigma_{u_{R}, t}^{2}$ as a function of its empirical counterpart 
$R Q_{t} / R V_{t}^{2}$. Namely, motivated by standard results on the asymptotic distribution of $\widetilde{R V}_{t}$, in order to model the dynamics of $\sigma_{u_{R}, t}^{2}$, letting $Y_{t}=\sqrt{R Q_{t}} / R V_{t}$, we have considered the following specification

$$
\sigma_{u_{R}, t}^{2}=\exp \left\{\delta_{0}+\delta_{1} V\left(Y_{t-1}\right)\right\},
$$

where the function $V($.$) has been chosen to be either the \log$ or the identity function; the exponential formulation guarantees the positivity of the estimated variance, thus avoiding to impose any constraints on the parameters $\delta_{0}$ and $\delta_{1}$. For $\delta_{1}=0$ the (E)TVRGARCH model is obtained as a special case.

Remark 2. As shown by Hansen et al. (2012) for the basic RGARCH model, the TVRGARCH model can be also represented as a Hidden Markov Model (HMM) driven by the latent chain $\widetilde{h}_{t}$. Namely, substituting the measurement equations for $\widetilde{R V} t$ and $\widetilde{R Q_{t}}$ into $\widetilde{h}_{t}$, we obtain the following representation of the conditional variance equation of the ETV-RGARCH model

$$
\widetilde{h}_{t}=\lambda_{0}+\lambda_{1, t} \widetilde{h}_{t-1}+\lambda_{2} \widetilde{h}_{t-1}^{2}+w_{t-1}^{*}
$$

where, under the stated assumptions, $w_{t}^{*}$ is a sequence of iid errors ${ }^{1}$ defined as

$$
w_{t}^{*}=\kappa_{3} w_{R, t}+\gamma_{1} \xi_{R} w_{Q, t}+\gamma_{1} w_{R, t} w_{Q, t}+\gamma_{2} w_{R, t}^{2}
$$

and

$$
\begin{aligned}
\lambda_{0} & =\omega+\gamma \xi_{R}+\gamma_{1} \xi_{R} \xi_{Q}+\gamma_{2} \xi_{R}^{2}, \\
\lambda_{1, t} & =\kappa_{0}+\kappa_{1} w_{R, t-1}+\kappa_{2} w_{Q, t-1}, \\
\lambda_{2} & =\beta_{1} \varphi_{Q}+\beta_{2} \varphi_{R}+\gamma_{1} \varphi_{R} \varphi_{Q}+\gamma_{2} \varphi_{R}^{2}, \\
\kappa_{0} & =\beta+\beta_{1} \xi_{Q}+\beta_{2} \xi_{R}+\gamma \varphi_{R}+\gamma_{1} \xi_{R} \varphi_{Q}+\gamma_{1} \xi_{Q} \varphi_{R}+2 \gamma_{2} \xi_{R} \varphi_{R}, \\
\kappa_{1} & =\beta_{2}+\gamma_{1} \varphi_{Q}+2 \gamma_{2} \varphi_{R}, \\
\kappa_{2} & =\beta_{1}+\gamma_{1} \varphi_{R} \\
\kappa_{3} & =\gamma+\gamma_{1} \xi_{Q}+2 \gamma_{2} \xi_{R} .
\end{aligned}
$$

The equivalent representation for TV-RGARCH models can be obtained by substituting $\gamma_{2}=-\gamma_{1}$ and $\beta_{2}=-\beta_{1}$ in the above equations. So, although both (E)TV-RGARCH and RGARCH models can be written as HMM models, the stochastic structure of the (E)TV-RGARCH model is more complex than that of the RGARCH model, since the model is now driven by a non-linear latent chain. This feature substantially complicates the derivation of stationarity and ergodicity conditions for the proposed TV-RGARCH models. Investigation of these problems goes beyond the scope of this paper and has been currently left for future research.

\footnotetext{
${ }^{1}$ Note that $E\left(w_{t}^{*}\right) \neq 0$. However, without any loss of generality, equation (32) could be written as

$$
\widetilde{h}_{t}=\bar{\lambda}_{0}+\lambda_{1, t} \widetilde{h}_{t-1}+\lambda_{2} \widetilde{h}_{t-1}^{2}+\bar{w}_{t-1},
$$

where $\bar{\lambda}_{0}=\lambda_{0}+E\left(w_{t}^{*}\right)$ and $\bar{w}_{t}=w_{t}^{*}-E\left(w_{t}^{*}\right)$, so that $E\left(\bar{w}_{t}\right)=0$. The value of $E\left(w_{t}^{*}\right)$ can be derived by simple algebra.
} 


\section{Estimation and inference}

The estimation of model parameters can be easily done by numerically maximizing the likelihood function implied by appropriate assumptions made on the model's error terms $z_{t}, u_{t, R}$ and $u_{t, Q}$. In particular, the joint conditional density of $\left(r_{t}, \widetilde{R V}, \widetilde{R Q}_{t}\right)$ can be factorized as

$$
f_{r, R, Q}\left(r_{t}, \widetilde{R V}_{t}, \widetilde{R Q}_{t} \mid \mathscr{F}_{t-1}\right)=f_{r}\left(r_{t} \mid \mathscr{F}_{t-1}\right) f_{R, Q}\left(\widetilde{R V}_{t}, \widetilde{R Q}_{t} \mid r_{t}, \mathscr{F}_{t-1}\right)
$$

It follows that the contribution of the $t$-th observation to the overall log-likelihood can be decomposed as

$$
\begin{aligned}
\ell\left(r_{t}, \widetilde{R V}_{t}, \widetilde{R Q}_{t} \mid \mathscr{F}_{t-1}, \theta\right) & =\log \left(f_{r, R, Q}\left(r_{t}, \widetilde{R V}_{t}, \widetilde{R Q}_{t} \mid \mathscr{F}_{t-1}\right)\right)=\log \left(f_{r}\left(r_{t} \mid \mathscr{F}_{t-1}\right)\right) \\
& +\log \left(f_{R, Q}\left(\widetilde{R V}_{t}, \widetilde{R Q}_{t} \mid r_{t} \mathscr{F}_{t-1}\right)\right) .
\end{aligned}
$$

Due to the normality assumption for $\mathbf{u}_{t}$ formulated in Section $5,\left(\widetilde{R V}_{t}, \widetilde{R Q}_{t} \mid r_{t}, \mathscr{F}_{t-1}\right)$ follows a $M V N_{2}$ distribution. Further, we assume $z_{t} \sim \sqrt{\frac{v-2}{v}} t_{v}$, where $t_{v}$ denotes a Student's t distribution with $v$ degrees of freedom. The overall log-likelihood will be then given by

$$
\begin{aligned}
\mathscr{L}(\mathbf{r}, \widetilde{\mathbf{R V}}, \widetilde{\mathbf{R Q}} \mid \theta) & =\sum_{t=1}^{T} \log \left(f_{r}\left(r_{t} \mid \mathscr{F}_{t-1}\right)\right)+\sum_{t=1}^{T} \log \left(f_{R, Q}\left(\widetilde{R V}_{t}, \widetilde{R Q_{t}} \mid r_{t}, \mathscr{F}_{t-1}\right)\right) \\
& =\ell_{r}+\ell_{R, Q} .
\end{aligned}
$$

We will refer to $\ell_{r}$ and $\ell_{R, Q}$ as the partial and measurement log-likelihoods respectively. Under the stated distributional assumptions, we get

$$
\begin{aligned}
\ell\left(r_{t}, \widetilde{R V}_{t}, \widetilde{R Q_{t}} \mid \mathscr{F}_{t-1}, \theta\right) & =\widetilde{K(v)}-\frac{1}{2} \widetilde{h}_{t}-\frac{v+1}{2} \log \left[1+\frac{r_{t}^{2}}{h_{t}(v-2)}\right] \\
& -\frac{1}{2} \log \left(\left|\Sigma_{\mathbf{u}}\right|\right)-\frac{1}{2} \mathbf{u}_{t}^{\prime} \Sigma_{\mathbf{u}}^{-1} \mathbf{u}_{t},
\end{aligned}
$$

for $t=1, \ldots, T$, where $\theta$ is the vector of unknown model parameters, $\mathbf{u}_{t}=\left(u_{t, R}, u_{t, Q}\right)^{\prime}$ and $\widetilde{K(v)}=\log (K(v))$, with

$$
K(v)=\frac{\Gamma\left(\frac{v+1}{2}\right)}{\sqrt{\pi(v-2)} \Gamma\left(\frac{v}{2}\right)} .
$$

The MLE of $\theta$ can be obtained by numerically maximizing the aggregated loglikelihood

$$
\hat{\theta}_{T}=\underset{\theta}{\operatorname{argmax}} \sum_{t=1}^{T} \mathscr{L}(\mathbf{r}, \widetilde{\mathbf{R V}}, \widetilde{\mathbf{R Q}} \mid \theta)
$$

Under the usual regularity conditions, standard errors for the elements of $\hat{\theta}_{T}$ can be easily obtained from the numerically approximated observed Fisher information matrix 
and inference can be performed relying on the asymptotic normality of $\hat{\theta}_{T}$. In order to double check the validity of the standard asymptotic results on the distribution of $\hat{\boldsymbol{\theta}}_{T}$, as in Borup and Jakobsen (2019), exploiting the dynamically complete nature of the proposed model, we have implemented a parametric Bootstrap resampling algorithm along the lines described in Paparoditis and Politis (2009). The main steps of the Bootstrap resampling procedure are summarized below. Throughout the presentation, the following notational conventions will be adopted: $X^{(B)}$ denotes the Bootstrap replicate of $X, \hat{\psi}$ is the estimate of coefficient $\psi$ based on in-sample data and, finally, the notation $\hat{\psi}^{(B)}$ denotes the estimate of coefficient $\psi$ based on bootstrapped data.

1. Save the residual vector from in-sample estimation

$$
\mathbf{e}_{t}=\left(\hat{z}_{t}, \hat{u}_{t, R}, \hat{u}_{t, Q}\right)^{\prime}
$$

and standardize it using the estimated variance and covariance matrix of $\mathbf{e}_{t}$

$$
\mathbf{a}_{t}=\hat{\Sigma}_{\mathbf{e}}^{-1 / 2} \mathbf{e}_{t}, \quad t=1, \ldots, T,
$$

where

$$
\hat{\Sigma}_{\mathbf{e}}=\left(\begin{array}{cc}
1 & \mathbf{0}_{1,2} \\
\mathbf{0}_{2,1} & \hat{\Sigma}_{\mathbf{u}}
\end{array}\right)
$$

with $A^{-1 / 2}$ denoting the Cholesky decomposition of the matrix $A$ and $\mathbf{0}_{r, s}$ being a $(r \times s)$ matrix of zeros.

2. Resample with replacement the time series of $\mathbf{a}_{t}$ to generate the time series of Bootstrap residuals $\mathbf{e}_{t}^{(B)}=\hat{\Sigma}_{\mathbf{e}}^{1 / 2} \mathbf{a}_{t}^{(B)}=\left(\hat{z}_{t}^{(B)}, \hat{u}_{t, R}^{(B)}, \hat{u}_{t, Q}^{(B)}\right)^{\prime}$.

3. Using the $\mathbf{e}_{t}^{(B)}$, recursively generate a Bootstrap replicate of $\left(r_{t}, R V_{t}, R Q_{t}\right)^{\prime}$ for $t=1, \ldots, T$. The set of recursions needed to generate $\left(r_{t}^{(B)}, R V_{t}^{(B)}, R Q_{t}^{(B)}\right)^{\prime}$ is given by

$$
\begin{aligned}
& \hat{\beta}_{t}^{(B)}=\hat{\beta}+\hat{\beta}_{1} \log \left(\sqrt{R Q_{t-1}^{(B)}} / R V_{t-1}^{(B)}\right)=\hat{\beta}+\hat{\beta}_{1} \widetilde{Y}_{t-1}^{(B)}, \\
& \hat{\gamma}_{t}^{(B)}=\hat{\gamma}+\hat{\gamma}_{1} \log \left(\sqrt{R Q_{t-1}^{(B)}} / R V_{t-1}^{(B)}\right)=\hat{\gamma}+\hat{\gamma}_{1} \widetilde{Y}_{t-1}^{(B)}, \\
& \widetilde{h}_{t}^{(B)}=\hat{\omega}+\hat{\gamma}_{t}^{(B)} \widetilde{R V}_{t-1}^{(B)}+\hat{\beta}_{t}^{(B)} \widetilde{h}_{t-1}^{(B)}, \\
& r_{t}^{(B)}=\sqrt{h_{t}^{(B)}} z_{t}^{(B)}, \\
& \mathbf{q}_{t}^{(B)}=\hat{\xi}+\hat{\varphi} \widetilde{h}_{t}^{(B)}+\hat{M}_{\tau} \zeta_{t}^{(B)}+\mathbf{u}_{t}^{(B)}, \\
& \mathbf{u}_{t}^{(B)}=\left(u_{t, R}^{(B)}, u_{t, Q}^{(B)}\right)^{\prime}, \zeta_{t}^{(B)}=\left(z_{t}^{(B)},\left(z_{t}^{(B)}\right)^{2}-1\right)^{\prime} \text { and } \\
& \hat{M}_{\tau}=\left(\begin{array}{ll}
\hat{\tau}_{1, R} & \hat{\tau}_{2, R} \\
\hat{\tau}_{1, Q} & \hat{\tau}_{2, Q}
\end{array}\right) .
\end{aligned}
$$


4. Fit the model to the Bootstrapped data $\left(r_{t}^{(B)}, \widetilde{R V}_{t}^{(B)}, \widetilde{R Q}_{t}^{(B)}\right)^{\prime}$ and save the estimated parameter vector $\hat{\theta}^{(B)}$.

5. Repeat steps (ii)-(iv) for $B=999$ times.

The resampling procedure described above is later used to provide an estimate of the sampling distributions of the estimated coefficients, along with standard errors and confidence intervals. The results of this exercise, discussed in detail in Section 7.2 , suggest that, overall, the asymptotic normality assumption deriving from standard likelihood theory provides a close approximation to the estimated distributions.

\section{Empirical application}

\subsection{Data}

In order to assess the merits of the proposed approach for risk management, we present the results of an empirical application to four major stock market indices: DAX 30 (Germany), FTSE 100 (UK), Hang Seng (Hong Kong) and S\&P 500 (USA). For each of these markets, 5-minute time series of the index value were downloaded from Thomson Reuters Tick History considering the period from January 2002 to April 2018. Daily open-to-close returns and realized measures were then computed limiting the attention to the official trading hours of each index. Furthermore, the data were cleaned removing the last day of each year, some extreme outliers and the last 5minute observation of each trading day, as usual. Due to the cleaning procedure, different trading days and holiday variations, the sample period consists of 4096 daily observations for DAX 30, 4063 for FTSE 100, 3951 for Hang Seng (HSI) and 4014 for S\&P 500.

Looking at the time plots of the daily open-to-close log-returns (Figure 1) and 5minute RVs (Figure 2), four important events can be detected. First, the effects of the 2008-2009 financial crisis are clearly visible in all the series, while the effects of the 2011-2012 sovereign debt crisis are more easily detectable in the US and European series, being particularly evident for the DAX series. Similarly, at the beginning of the sample period, a high volatility period, mainly related to the explosion of the dotcom bubble and the introduction of Euro, is mostly visible for the DAX and FTSE indices, less clearly evident in the S\&P 500 and not detectable in the HSI index. Last, a high volatility period affects, with different intensities, all the markets across 2015 and 2016. Different events can be identified as potential determinants of this phenomenon: including the Chinese stock market turbulence; the Greek debt default in 2015; the end of quantitative easing in the United States at the end of 2014; and the Brexit referendum in 2016. The main descriptive statistics of returns are reported in Table 15 in the Empirical Appendix.

In line with asymptotic theory, a point measure of the accuracy of the logtransformed realized variance is obtained by computing the ratio $Y_{t}$. The time series plots in Figure 3 reveal that, for all markets considered, the log-ratio $\widetilde{Y}_{t}$ is characterized by remarkable short term fluctuations, thus supporting the intuition that accounting 
Figure 1: Time series of daily open-to-close log-returns
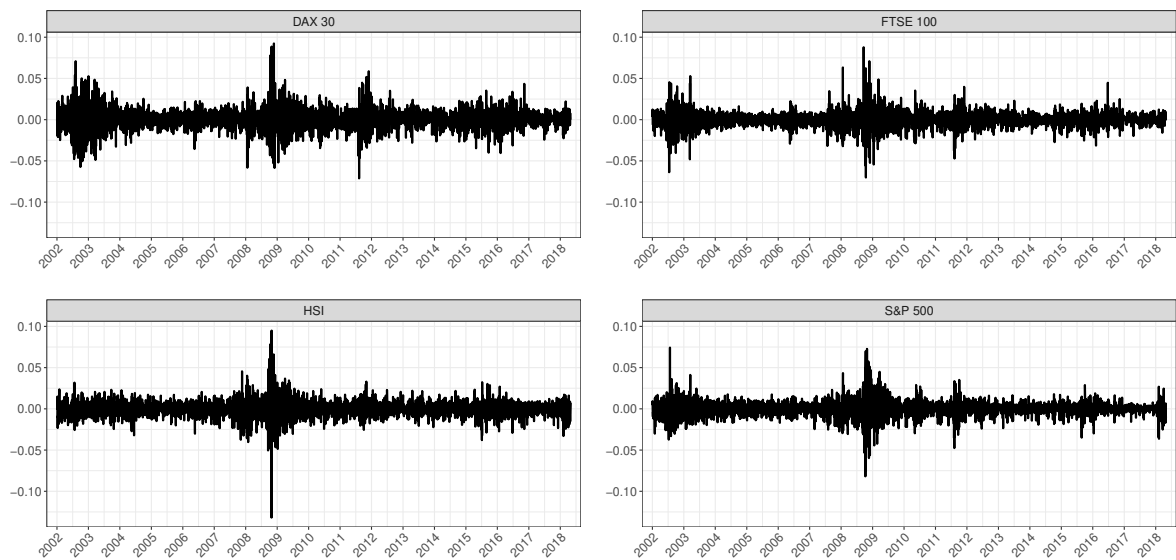

Daily log-returns for the stock market indices DAX 30 (top-left), FTSE 100 (top-right), Hang Seng (bottomleft) and S\&P500 (bottom-right) for the full sample period 2002/01/01 - 2018/04/30.

for a time-varying attenuation bias effect could be beneficial for volatility and risk forecasting.

\subsection{In-sample analysis}

Referring to the four stock market indices considered, this section assesses the insample performance of the proposed models, taking the standard RGARCH model as a benchmark and considering the full sample period from 2002/01/01 to 2018/04/30. Model parameters have been estimated by maximum likelihood as described in Section 6. Table 2 reports the estimated coefficients and standard errors, based on the observed information matrix. Overall, the fitted coefficients are in most cases significantly different from 0 at the usual 5\% level. The only exceptions are the intercepts of the volatility, $\omega$, and measurement equations, $\xi_{R}$ and $\xi_{Q}$, that, in some cases, result to be not significantly different from 0 . Also, for DAX 30 and FTSE 100, the $\beta$ coefficient is not significant for the ETV-RGARCH. Similarly, the $\tau_{1, R}$ and $\tau_{1, Q}$ coefficients are never significant for the HSI index. The $\xi_{R}$ and $\varphi_{R}$ coefficients are, overall, very close to 0 and 1 , respectively, suggesting that the log-transformed $R V$ is an approximately unbiased proxy of the latent $\log \left(h_{t}\right)$.

Focusing on the TV-RGARCH models, it is interesting to see that the estimated $\gamma_{1}$ and $\beta_{1}$ coefficients are significantly different from 0 at the usual 5\% level, providing evidence in favor of the presence of time-varying attenuation bias effects. Also, as expected, $\gamma_{1}$ and $\beta_{1}$ have negative and positive signs, respectively, confirming the intuition that, when $\log \left(R V_{t-1}\right)$ provides a more (less) accurate estimate of the latent signal $\log \left(I V_{t}\right)$, the following hold: i) the impact of $\widetilde{R V}_{t-1}$ on $\widetilde{h}_{t}$, as measured by $\gamma_{t}$, is higher (lower); ii) the contribution of the inertial component $\log \left(h_{t-1}\right)$ to the value of $\log \left(h_{t}\right)$, as measured by $\beta_{t}$, is lower (higher). This behavior is clearly evident 
Table 2: In-sample estimation results

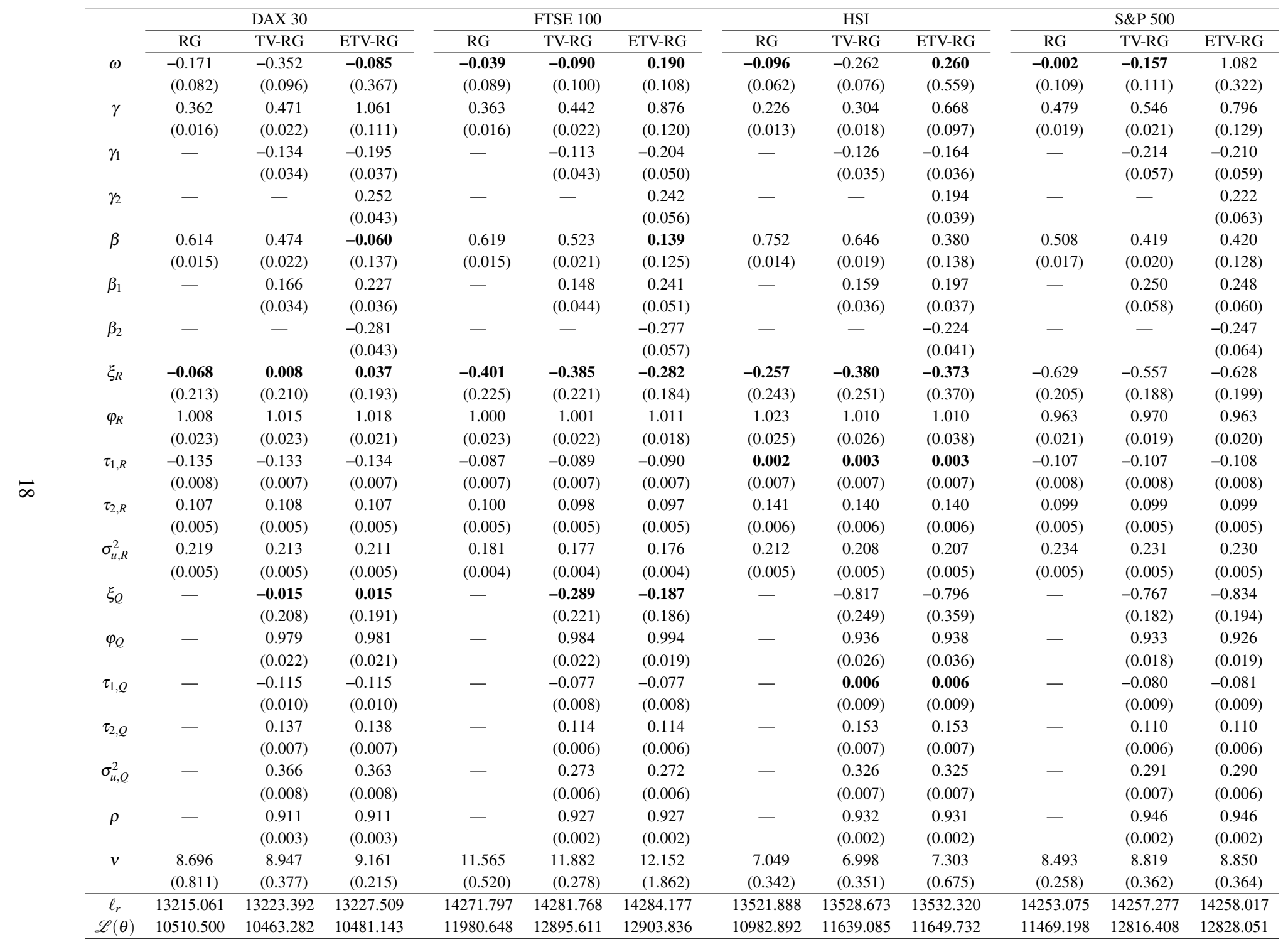

In-sample parameter estimates for the full sample period 2002/01/01 - 2018/04/30. $\ell_{r}$ : partial log-likelihood. $\mathscr{L}(\theta)$ : log-likelihood. Standard errors are reported in parentheses. Parameters that are not significant at the $5 \%$ level are reported in boldface. 
Figure 2: Time series of 5-min Realized Volatility
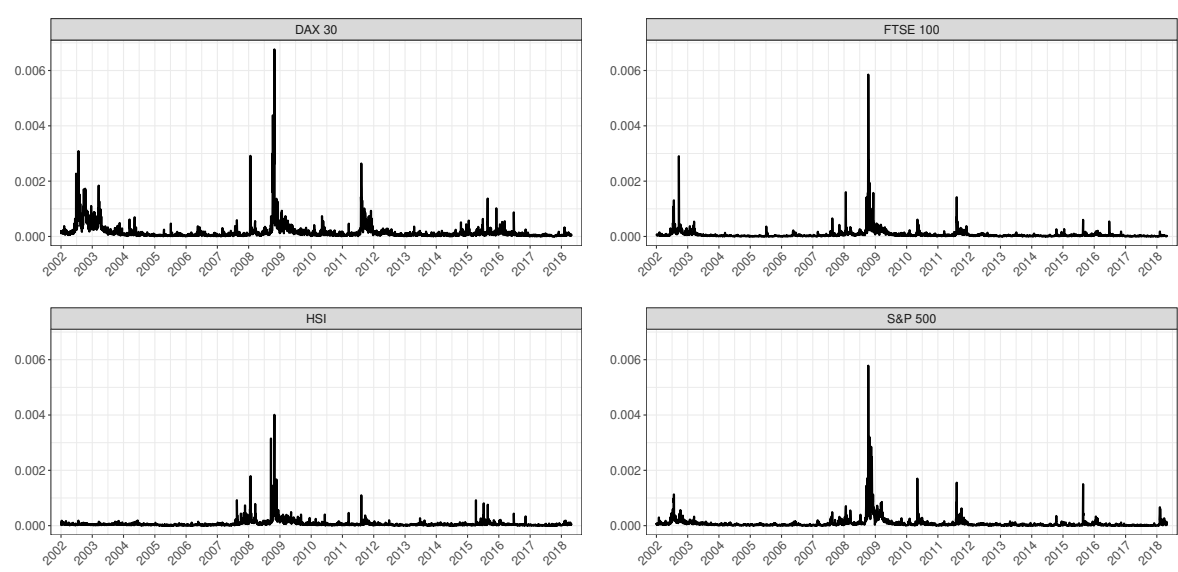

Daily 5-minute Realized Volatility for the stock market indices DAX 30 (top-left), FTSE 100 (top-right), Hang Seng (bottom-left) and S\&P500 (bottom-right) for the full sample period 2002/01/01 - 2018/04/30.

from Figure 4, representing the time series of the fitted $\gamma_{t}$ and $\beta_{t}$ coefficients for the TV-RGARCH model. Furthermore, the plots reveal another interesting feature: the average of the fitted $\gamma_{t}$ (dashed line in the plot) is substantially higher than the fitted time-invariant $\gamma$ coefficient of the standard RGARCH model (solid line in the plot). A similar argument holds, reversed, for $\beta_{t}$. This implies that the volatility and tail risk estimates generated by the fitted RGARCH and TV-RGARCH models will differ due to the action of two different factors: the first is a level effect related to the discrepancy between $E\left(\gamma_{t}\right)$ and $E\left(\beta_{t}\right)$, on one side, and the RGARCH parameters $\gamma$ and $\beta$, on the other; differently, the second factor depends on short term fluctuations of the ratio $Y_{t}$ around its mean level.

The same general picture applies to the ETV-RGARCH model, with the estimated $\gamma_{1}$ and $\beta_{1}$ being negative and positive, respectively, as for the TV-RGARCH model. Further, as expected, $\gamma_{2}$ and $\beta_{2}$ take opposite signs. The dynamic profiles of the time-varying coefficients $\gamma_{t}$ and $\beta_{t}$ (Figure 5) are qualitatively not different from that observed for the TV-RGARCH model. This is confirmed by Table 3, showing the sample correlation coefficients between $\gamma_{t}$ and $\beta_{t}$, fitted by TV-RGARCH and ETVRGARCH respectively.

Table 4 reports the results of three sets of likelihood ratio tests. First, we separately test the validity of the restrictions implied by the standard RGARCH models against the alternative TV-RGARCH and ETV-RGARCH models. Second, we test the TVRGARCH hypothesis against the alternative of a more general ETV-RGARCH model. In the first case, the reference asymptotic distribution of the test statistic under the null is given by a $\chi_{2}^{2}$, for the TV-RGARCH model, and by a $\chi_{4}^{2}$, if the alternative corresponds to an ETV-RGARCH model. In the second set, the reference distribution for testing the TV-RGARCH model against a more general ETV-RGARCH is given by a $\chi_{2}^{2}$ distribution. In both cases, since the full-likelihoods of RGARCH and (E)TV- 
Figure 3: Time series of $\log \left(\frac{\sqrt{R Q_{t}}}{R V_{t}}\right)$
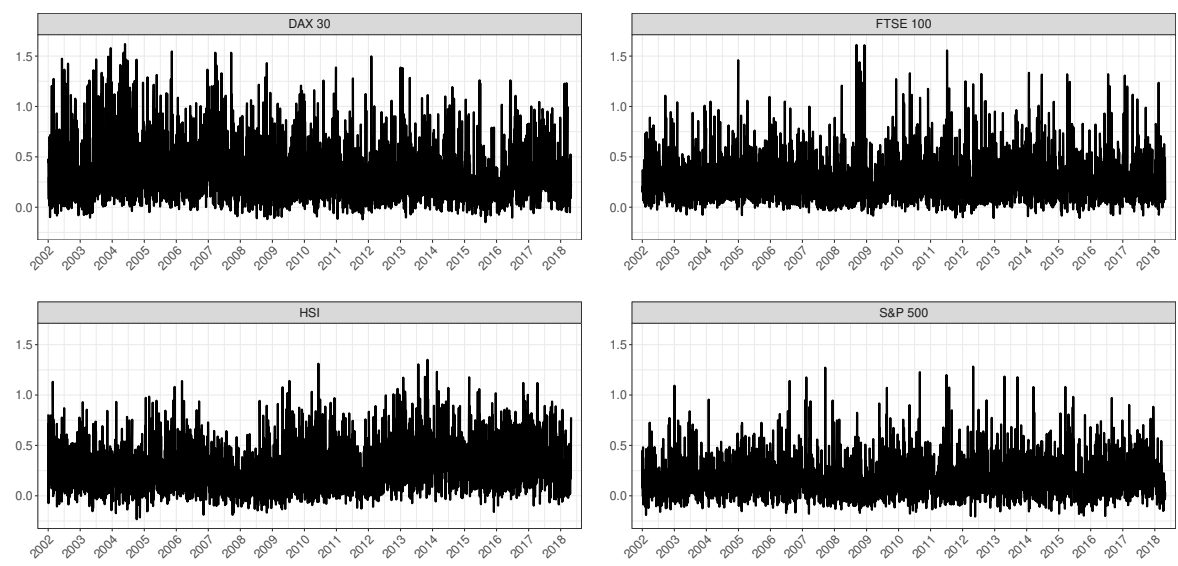

Daily $\log$-ratio $\log \left(\sqrt{R Q_{t}} / R V_{t}\right)$ for the stock market indices DAX 30 (top-left), FTSE 100 (top-right), Hang Seng (bottom-left) and S\&P500 (bottom-right) for the full sample period 2002/01/01 - 2018/04/30.

RGARCH are based on different information sets, testing is based on the partial loglikelihood component $\ell_{r}$. For all the markets considered, the benchmark RGARCH model is always rejected at the usual 5\% significance level against both alternatives: TV-RGARCH and ETV-RGARCH. When testing the TV-RGARCH against the more general ETV-RGARCH, the data provide mixed evidence, since we find that only in two cases out of four, DAX 30 and HSI, the null is rejected. The last set of likelihood ratio tests, in the bottom panel of Table 4, again compares the TV-RGARCH model, under the null, against the alternative hypothesis of an ETV-RGARCH but using the full likelihood $\mathscr{L}(\theta)$. The results show that, when considering the full likelihood, the TV-RGARCH model is always rejected against the more flexible ETV-RGARCH model.

Table 3: Sample correlation coefficients between $\gamma_{t}\left(\rho_{\gamma}\right)$ and $\beta_{t}\left(\rho_{\beta}\right)$ fitted by TVRGARCH and ETV-RGARCH models.

\begin{tabular}{ccccc}
\hline & DAX 30 & FTSE 100 & HSI & S\&P 500 \\
\hline$\rho_{\gamma}$ & 0.640 & 0.722 & 0.811 & 0.952 \\
$\rho_{\beta}$ & 0.720 & 0.799 & 0.892 & 1.000 \\
\hline
\end{tabular}

As a robustness check, we also consider the estimation of heteroskedastic variants of the TV-RGARCH and ETV-RGARCH, as described in Remark 1 at the end of Section 5. Our data, however, do not provide strong evidence in favor of the presence of heteroskedasticity, for both the conditional variance specifications considered. Namely, the estimation results for heteroskedastic models, reported in Table 18 in the Empirical Appendix, suggest that the homoskedasticity assumption (corresponding 
Figure 4: Time series of estimated $\gamma_{t}$ and $\beta_{t}$ for TV-RGARCH
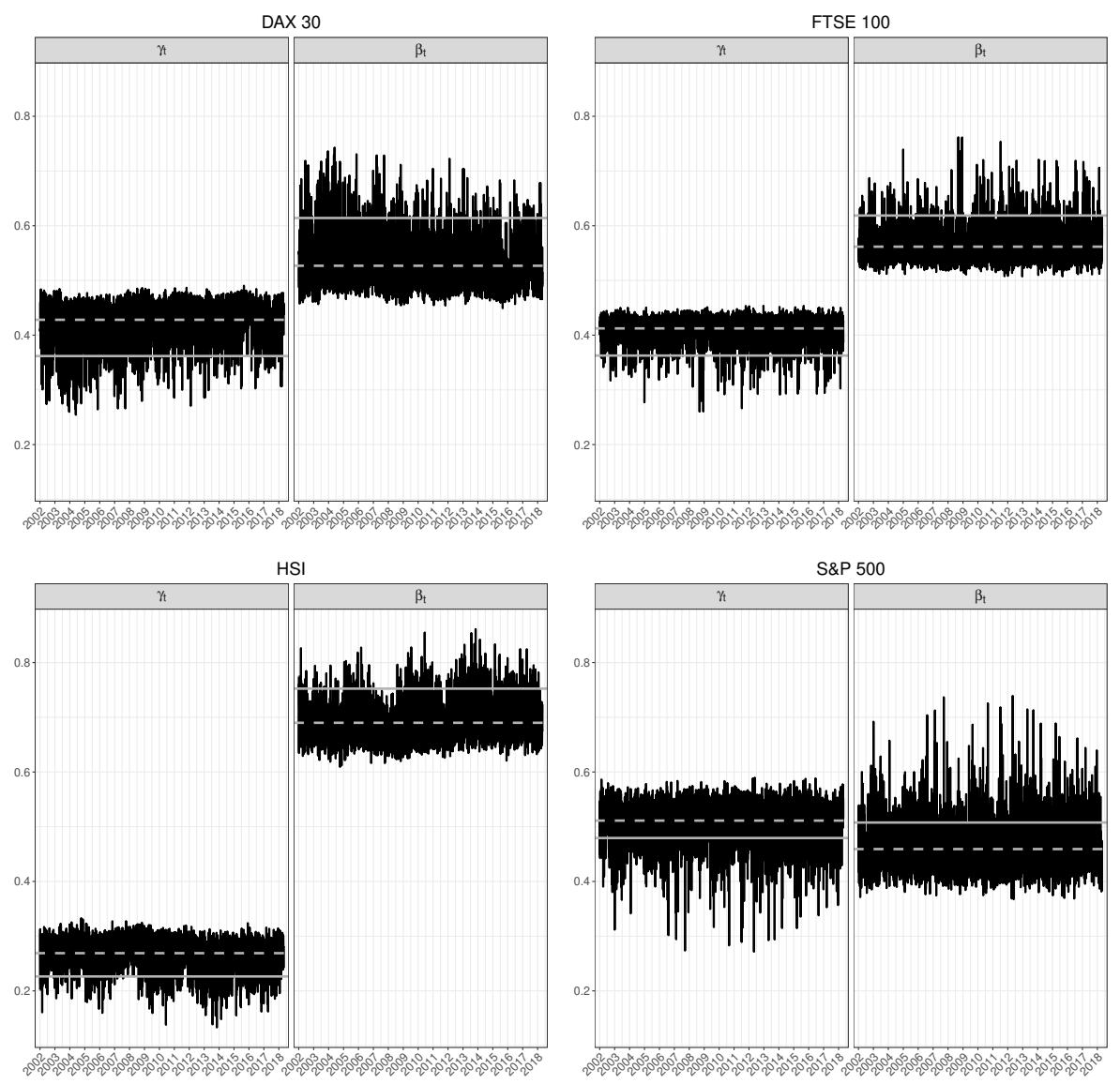

Time-varying $\gamma_{t}$ and $\beta_{t}$ of TV-RGARCH model for the stock market indices DAX 30 (top-left), FTSE 100 (top-right), Hang Seng (bottom-left) and S\&P500 (bottom-right) for the full sample period 2002/01/01 2018/04/30. Gray solid-line: RGARCH coefficient. Gray dashed-line: average of TV-RGARCH timevarying coefficient. 
Figure 5: Time series of estimated $\gamma_{t}$ and $\beta_{t}$ for ETV-RGARCH
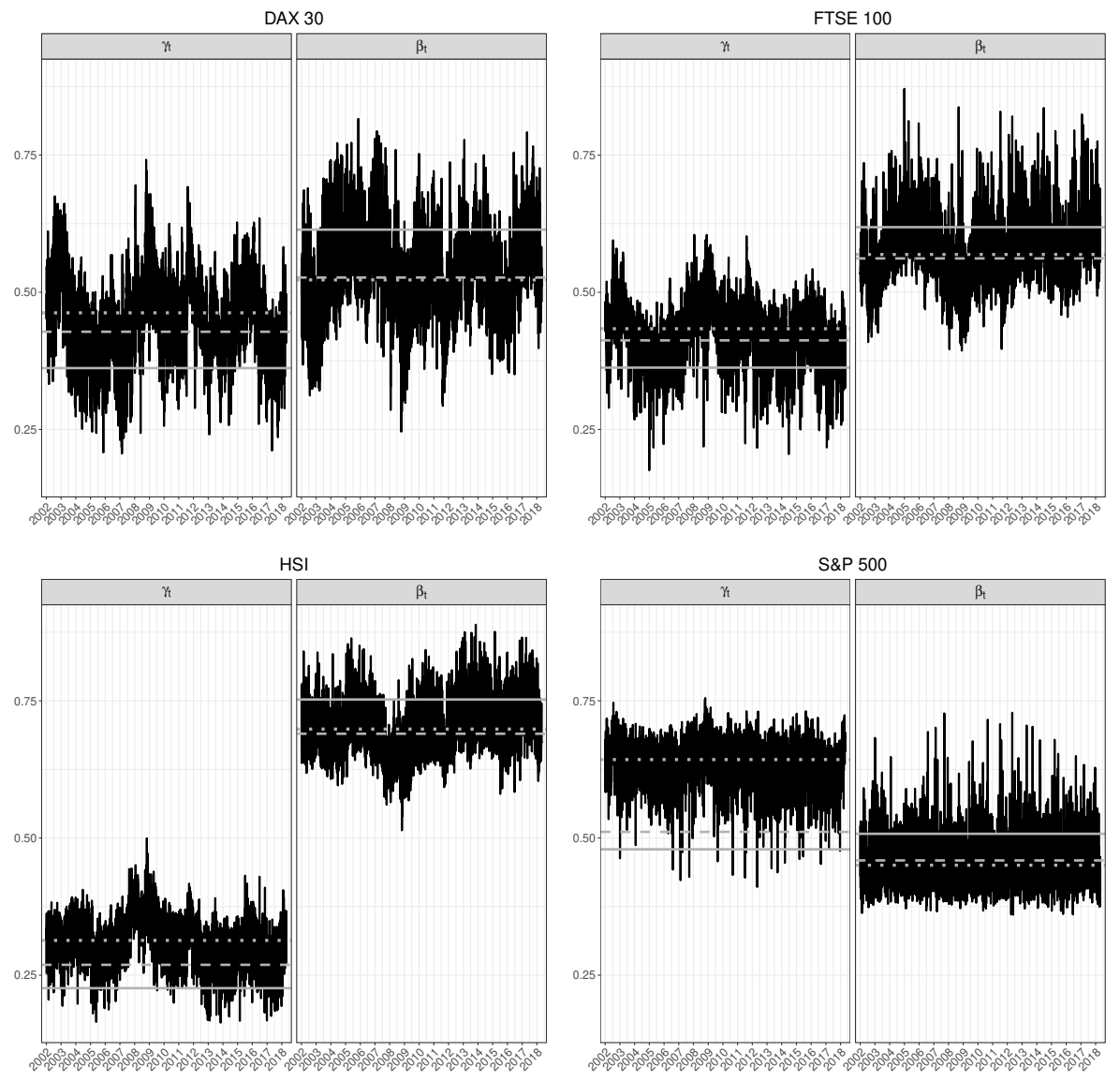

Time-varying $\gamma_{t}$ and $\beta_{t}$ of ETV-RGARCH model for the stock market indices DAX 30 (top-left), FTSE 100 (top-right), Hang Seng (bottom-left) and S\&P500 (bottom-right) for the full sample period 2002/01/01 - 2018/04/30. Gray solid-line: RGARCH coefficient. Gray dashed-line: average of TV-RGARCH timevarying coefficient. Gray dotted-line: average of ETV-RGARCH time-varying coefficient. 
Table 4: Likelihood ratio statistics for the full sample period 2002/01/01 - 2018/04/30. Top panel: likelihood ratio statistics for the partial $\log$-likelihood $\ell_{r}$. Bottom panel: likelihood ratio statistics for the full $\log$-likelihood $\mathscr{L}(\theta)$. P-values are reported in parentheses.

\begin{tabular}{|c|c|c|c|c|c|c|c|c|}
\hline & \multicolumn{2}{|c|}{ DAX 30} & \multicolumn{2}{|c|}{ FTSE 100} & \multicolumn{2}{|c|}{ HSI } & \multicolumn{2}{|c|}{ S\&P 500} \\
\hline & TV-RG & ETV-RG & TV-RG & ETV-RG & TV-RG & ETV-RG & TV-RG & ETV-RG \\
\hline \multirow[t]{2}{*}{ RG } & 16.663 & 24.896 & 19.941 & 24.760 & 13.570 & 20.864 & 8.404 & 9.890 \\
\hline & $(0.000)$ & $(0.000)$ & $(0.000)$ & $(0.000)$ & $(0.001)$ & $(0.000)$ & $(0.015)$ & $(0.042)$ \\
\hline \multirow[t]{2}{*}{ TV-RG } & - & 8.233 & - & 4.818 & - & 7.295 & - & 1.481 \\
\hline & & $(0.016)$ & & $(0.090)$ & & $(0.026)$ & & $(0.477)$ \\
\hline \multirow[t]{2}{*}{ TV-RG } & - & 35.722 & - & 16.450 & - & 21.294 & - & 23.286 \\
\hline & & $(0.000)$ & & $(0.000)$ & & $(0.000)$ & & $(0.000)$ \\
\hline
\end{tabular}

to $\delta_{1}=0$ ) cannot be rejected in the majority of cases. Also, the introduction of the heteroskedastic component does not seem to have a remarkable impact on the estimates of the other model parameters. Accordingly, a simple likelihood ratio test, comparing heteroskedastic vs homoskedastic models, would reveal that the introduction of the heteroskedastic component, in the vast majority of cases, does not bring to any significant improvement in the overall likelihood $\mathscr{L}$. Finally, we focused our attention on the partial likelihood $\ell_{r}$ which measures the ability of the fitted model to reproduce the conditional distribution of returns, hence being a the critical entity for risk management applications. Our findings indeed show that increments in $\ell_{r}$, when present, are always negligible. So, the introduction of the heteroskedastic component is not expected to bring any noticeable gains for tail risk forecasting. It is worth noting that, under this respect, our findings are in line with those of and Hansen et al. (2012) and Hansen and Huang (2016).

The above results are based on standard maximum likelihood theory. In order to double check the validity of the implied asymptotic approximation, we have implemented the Bootstrap resampling procedure described in Section 6 for all the model specifications considered. However, in order to save space, in this section we only report results for the ETV-RGARCH model. The results obtained for the RGARCH and TV-RGARCH models, qualitatively similar to those reported for the ETV-RGARCH, have been reported in the Empirical Appendix.

Figure 6 reports the histograms of the standardized Bootstrap estimates for the ETV-RGARCH model's parameters. In general, the plots suggest that the empirical distributions of the estimates are consistent with the asymptotic normality assumption. Mild positive skewness is detected only for the estimated degrees of freedom parameter $v$. Furthermore, Table 5 shows that the Bootstrap means and standard errors are in general very close to the ML estimated coefficients and associated asymptotic standard errors. In addition, the table also reports the $95 \%$ Bootstrap percentile confidence intervals for each of the estimated coefficients. Looking at the estimated intervals it can be immediately noted how the assessment of the significance of estimated coefficients 
based on asymptotic theory is in close agreement with the findings deriving from the analysis of the Bootstrap intervals.

\subsection{Out-of-sample analysis}

In this section the proposed model specifications are used to generate out-of-sample one-step-ahead forecasts of volatility, VaR and ES. Our forecasting design is based on a rolling window scheme with daily re-estimation. For all markets, the initial in-sample period covers the time interval from 2002/01/01 to 2008/05/31, resulting in different time series lengths for the different indices considered: 1604 for the DAX 30, 1590 for the FTSE 100, 1555 for the HSI and 1558 for the S\&P 500. For each index, subsequent re-estimations are then based over moving windows of the same length. The out-ofsample period has been chosen to allow the inclusion of the most relevant financial events of the current century, starting from the climax of the 2008-2009 financial crisis, while still keeping a sufficiently long in-sample estimation window.

The performances of the proposed models are compared with those of the standard RGARCH, taken as a benchmark. Also, as a further robustness check, we consider a set of alternative specifications of TV-RGARCH models characterized by different specifications of the time-varying coefficients $\beta_{t}$ and $\gamma_{t}$. These have been summarized in Table 6. The aim is here to double check the appropriateness of the specifications of $\gamma_{t}$ and $\beta_{t}$ discussed in Section 5 and the sensitivity of our empirical results to the model assumed for $\gamma_{t}$ and $\beta_{t}$.

Next, we assess the out-of-sample forecasting ability of the model considering different loss functions. First, the ability to accurately forecast the distribution of future returns, for each model, is assessed by computing, as in Hansen et al. (2012), the outof-sample predictive partial log-likelihood

$$
\hat{\ell}_{r}(\hat{\theta})_{t+1}=\widetilde{K(\hat{v})}-\frac{1}{2} \log \left(\hat{h}_{t+1}\right)-\frac{\hat{v}+1}{2} \log \left[1+\frac{r_{t+1}^{2}}{\hat{h}_{t+1}(\hat{v}-2)}\right],
$$

for $t=T, \ldots, T+H-1$, with $H$ being the length of the out-of-sample forecasting period.

The accuracy in forecasting future volatility is then evaluated by means of the QLIKE loss function. This choice is motivated by two considerations. First, the QLIKE is robust to noisy volatility proxies (Patton, 2011). Second, compared to other robust alternatives, this loss function has been found to be more powerful in rejecting poorly performing predictors (Liu et al., 2015). The QLIKE loss has been computed according to the formula

$$
\text { QLIKE }=\frac{1}{H} \sum_{j=1}^{H}\left(\log \left(\hat{h}_{T+j}\right)+\frac{R V_{T+j}}{\hat{h}_{T+j}}\right),
$$

where $\hat{h}_{T+j}$ is the 1-step-ahead conditional variance forecast at time $T+j$. It is trivial to show that models providing better forecasts will be characterized by lower values of QLIKE.

Furthermore, the quality of individual VaR forecasts is assessed using the Conditional Coverage test of Christoffersen (1998) and the Dynamic Quantile test of 
Figure 6: Histograms and superimposed non-parametric densities of 999 standardized Bootstrap estimates of the parameters of the ETV-RGARCH model
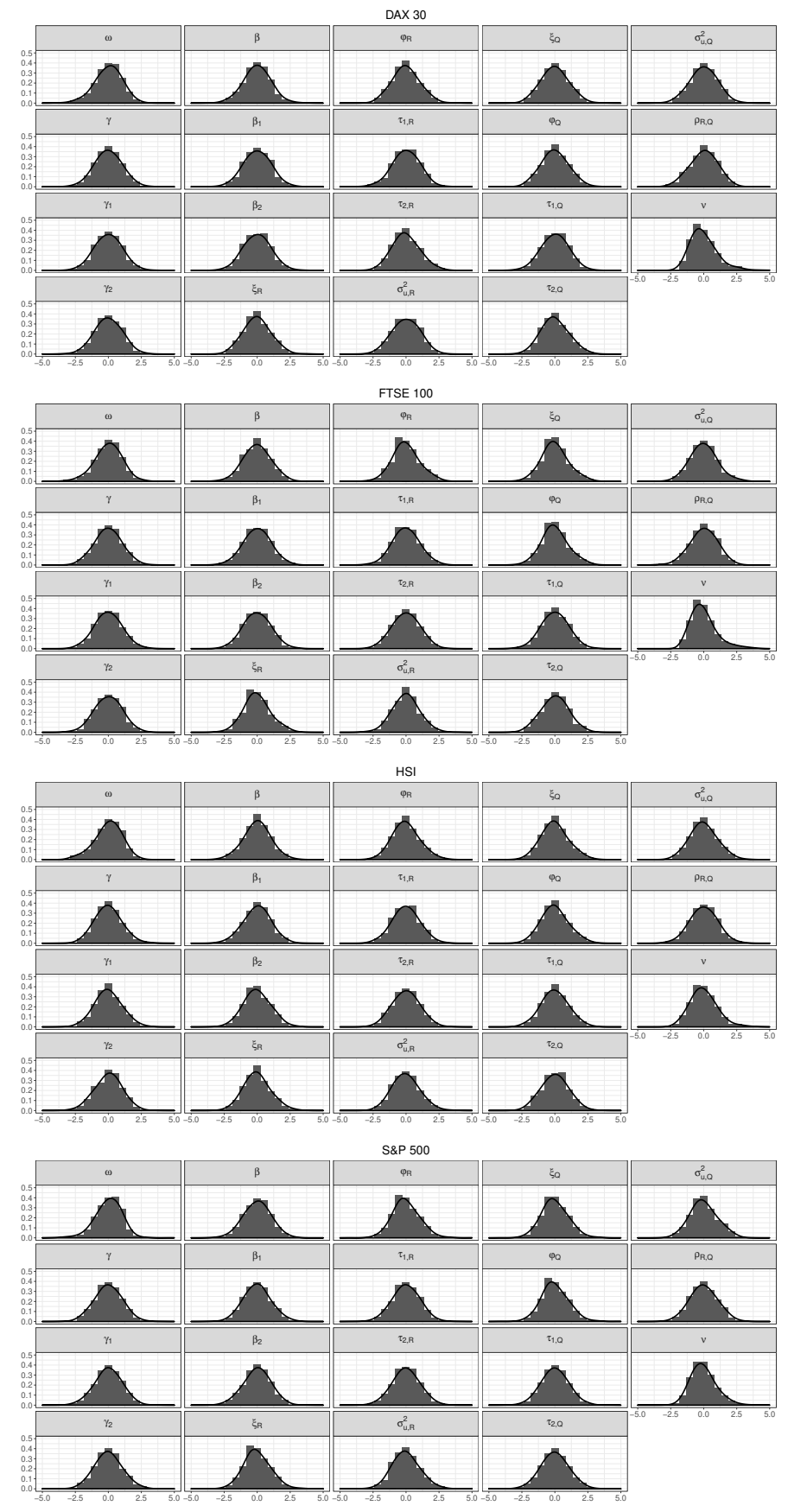
Table 5: Summary of Bootstrap estimates for the ETV-RGARCH model

\begin{tabular}{|c|c|c|c|c|c|c|c|c|c|c|c|c|c|c|c|c|c|c|c|c|c|c|c|c|}
\hline & \multicolumn{6}{|c|}{ DAX 30} & \multicolumn{6}{|c|}{ FTSE 100} & \multicolumn{6}{|c|}{ HSI } & \multicolumn{6}{|c|}{ S\&P 500} \\
\hline & Coef & $\mu_{B}$ & $\mathrm{SE}$ & SE.B & $q_{0.025}$ & $q_{0.975}$ & Coef & $\mu_{B}$ & $\mathrm{SE}$ & SE.B & $q_{0.025}$ & $q_{0.975}$ & Coef & $\mu_{B}$ & SE & SE.B & $q_{0.025}$ & $q_{0.975}$ & Coef & $\mu_{B}$ & SE & SE.B & $q_{0.025}$ & $q_{0.975}$ \\
\hline$\omega$ & -0.085 & -0.168 & 0.367 & 0.402 & -1.049 & 0.551 & 0.190 & 0.121 & 0.108 & 0.374 & -0.682 & 0.805 & 0.260 & 0.175 & 0.559 & 0.497 & -0.983 & 1.051 & 1.082 & 0.822 & 0.322 & 0.340 & 0.078 & 1.360 \\
\hline$\gamma$ & 1.061 & 1.056 & 0.111 & 0.104 & 0.854 & 1.256 & 0.876 & 0.870 & 0.120 & 0.121 & 0.638 & 1.099 & 0.668 & 0.667 & 0.097 & 0.106 & 0.469 & 0.880 & 0.796 & 0.779 & 0.129 & 0.125 & 0.535 & 1.019 \\
\hline$\gamma_{1}$ & 0.195 & -0.194 & 0.037 & 0.036 & -0.267 & -0.122 & -0.204 & -0.203 & 0.050 & 0.044 & -0.291 & -0.113 & -0.164 & -0.165 & 0.036 & 0.038 & -0.240 & -0.087 & -0.210 & -0.211 & 0.059 & 0.062 & -0.341 & -0.095 \\
\hline$\gamma_{2}$ & 0.252 & 0.251 & 0.043 & 0.040 & 0.172 & 0.328 & 0.242 & 0.241 & 0.056 & 0.048 & 0.151 & 0.334 & 0.194 & 0.197 & 0.039 & 0.042 & 0.114 & 0.276 & 0.222 & 0.224 & 0.063 & 0.064 & 0.098 & 0.357 \\
\hline$\beta$ & -0.060 & -0.072 & 0.137 & 0.111 & -0.302 & 0.153 & 0.139 & 0.133 & 0.125 & 0.125 & -0.106 & 0.373 & 0.380 & 0.365 & 0.138 & 0.119 & 0.120 & 0.604 & 0.420 & 0.387 & 0.128 & 0.129 & 0.129 & 0.631 \\
\hline$\beta_{1}$ & 0.227 & 0.226 & 0.036 & 0.036 & 0.154 & 0.297 & 0.241 & 0.240 & 0.051 & 0.045 & 0.150 & 0.330 & 0.197 & 0.199 & 0.037 & 0.040 & 0.118 & 0.276 & 0.248 & 0.248 & 0.060 & 0.062 & 0.131 & 0.379 \\
\hline$\beta_{2}$ & 0.281 & -0.282 & 0.043 & 0.040 & -0.359 & -0.203 & -0.277 & -0.277 & 0.057 & 0.050 & -0.372 & -0.183 & -0.224 & -0.227 & 0.041 & 0.044 & -0.313 & -0.139 & -0.247 & -0.251 & 0.064 & 0.065 & -0.382 & -0.123 \\
\hline$\xi_{R}$ & 0.037 & 0.054 & 0.193 & 0.279 & -0.463 & 0.619 & -0.282 & -0.264 & 0.184 & 0.289 & -0.791 & 0.357 & -0.373 & -0.350 & 0.370 & 0.361 & -1.021 & 0.391 & -0.628 & -0.615 & 0.199 & 0.250 & -1.073 & -0.116 \\
\hline$\varphi_{R}$ & 1.018 & 1.020 & 0.021 & 0.030 & 0.964 & 1.081 & 1.011 & 1.013 & 0.018 & 0.029 & 0.959 & 1.075 & 1.010 & 1.013 & 0.038 & 0.037 & 0.942 & 1.089 & 0.963 & 0.964 & 0.020 & 0.025 & 0.919 & 1.015 \\
\hline$\tau_{1, R}$ & -0.134 & -0.133 & 0.007 & 0.008 & -0.150 & -0.119 & -0.090 & -0.090 & 0.007 & 0.007 & -0.103 & -0.077 & 0.003 & 0.002 & 0.007 & 0.008 & -0.013 & 0.017 & -0.108 & -0.108 & 0.008 & 0.008 & -0.123 & -0.094 \\
\hline$\tau_{2, R}$ & 0.107 & 0.109 & 0.005 & 0.007 & 0.096 & 0.124 & 0.097 & 0.097 & 0.005 & 0.006 & 0.085 & 0.108 & 0.140 & 0.140 & 0.006 & 0.006 & 0.130 & 0.151 & 0.099 & 0.099 & 0.005 & 0.006 & 0.088 & 0.111 \\
\hline$\sigma_{u, R}^{2}$ & 0.211 & 0.211 & 0.005 & 0.005 & 0.200 & 0.221 & 0.176 & 0.175 & 0.004 & 0.005 & 0.165 & 0.186 & 0.207 & 0.206 & 0.005 & 0.007 & 0.194 & 0.220 & 0.230 & 0.229 & 0.005 & 0.006 & 0.218 & 0.241 \\
\hline$\xi_{Q}$ & 0.015 & 0.030 & 0.191 & 0.269 & -0.474 & 0.566 & -0.187 & -0.170 & 0.186 & 0.286 & -0.701 & 0.445 & -0.796 & -0.777 & 0.359 & 0.343 & -1.412 & -0.081 & -0.834 & -0.824 & 0.194 & 0.243 & -1.271 & -0.337 \\
\hline$\varphi_{Q}$ & 0.981 & 0.983 & 0.021 & 0.029 & 0.929 & 1.040 & 0.994 & 0.996 & 0.019 & 0.029 & 0.943 & 1.058 & 0.938 & 0.940 & 0.036 & 0.035 & 0.874 & 1.012 & 0.926 & 0.927 & 0.019 & 0.024 & 0.882 & 0.976 \\
\hline$\tau_{1, Q}$ & -0.115 & -0.115 & 0.010 & 0.012 & -0.138 & -0.093 & -0.077 & -0.077 & 0.008 & 0.009 & -0.094 & -0.061 & 0.006 & 0.005 & 0.009 & 0.010 & -0.014 & 0.026 & -0.081 & -0.081 & 0.009 & 0.010 & -0.099 & -0.061 \\
\hline$\tau_{2, Q}$ & 0.138 & 0.141 & 0.007 & 0.011 & 0.120 & 0.164 & 0.114 & 0.114 & 0.006 & 0.007 & 0.100 & 0.128 & 0.153 & 0.153 & 0.007 & 0.007 & 0.140 & 0.166 & 0.110 & 0.110 & 0.006 & 0.007 & 0.096 & 0.125 \\
\hline$\sigma_{u, Q}^{2}$ & 0.363 & 0.362 & 0.008 & 0.010 & 0.342 & 0.382 & 0.272 & 0.271 & 0.006 & 0.009 & 0.254 & 0.291 & 0.325 & 0.324 & 0.007 & 0.010 & 0.305 & 0.345 & 0.290 & 0.289 & 0.006 & 0.008 & 0.274 & 0.305 \\
\hline$\rho$ & 0.911 & 0.911 & 0.003 & 0.003 & 0.905 & 0.916 & 0.927 & 0.927 & 0.002 & 0.003 & 0.921 & & 0.931 & 0.931 & 0.002 & 0.002 & 0.927 & 0.9 & 0.946 & 0.946 & 0.002 & 0.002 & 0.942 & 1.950 \\
\hline & 9.161 & 9.257 & 0.215 & 1.430 & 7.136 & 12.820 & 12.152 & 12.568 & 1.862 & 2.365 & 9.179 & 18.831 & 7.303 & 7.399 & 0.675 & 0.789 & 6.013 & 9.220 & 8.850 & 9.042 & 0.364 & 1.239 & 7.104 & 11.717 \\
\hline
\end{tabular}

Coef: estimated coefficient; $\mu_{B}$ : mean of Bootstrap estimates; SE: asymptotic standard error ; SE.B: standard error of Bootstrap estimates; $q_{0.025}: 2.5 \%$ Bootstrap percentile; $q_{0.975}: 97.5 \%$ Bootstrap percentile. 
Table 6: Model specifications for robustness check

\begin{tabular}{llll}
\hline \multicolumn{1}{c}{ Model } & \multicolumn{1}{c}{$\beta_{t}$ equation } & \multicolumn{1}{c}{$\gamma_{t}$ equation } & Error distribution \\
\hline TV-RGARCH $^{*}$ & $\beta_{t}=\beta+\beta_{1}\left(Y_{t-1}\right)$ & $\gamma_{t}=\gamma+\gamma_{1}\left(Y_{t-1}\right)$ & $\mathbf{u}_{t} \sim M V N_{2}\left(0, \Sigma_{\mathbf{u}}\right)$ \\
TV-RGARCH $^{*} \mathrm{~S}$ & $\beta_{t}=\beta+\beta_{1}\left(\sqrt{Y_{t-1}}\right)$ & $\gamma_{t}=\gamma+\gamma_{1}\left(\sqrt{Y_{t-1}}\right)$ & $\mathbf{u}_{t} \sim M V N_{2}\left(0, \Sigma_{\mathbf{u}}\right)$ \\
TV-RGARCH $^{*} \mathrm{~S} 2$ & $\beta_{t}=\beta+\beta_{1}\left(Y_{t-1}\right)^{2}$ & $\gamma_{t}=\gamma+\gamma_{1}\left(Y_{t-1}\right)^{2}$ & $\mathbf{u}_{t} \sim M V N_{2}\left(0, \Sigma_{\mathbf{u}}\right)$ \\
TV-RGARCH $^{*}-2$ & $\beta_{t}=\beta+\beta_{1}\left(Y_{t-1}\right)^{\beta_{2}}$ & $\gamma_{t}=\gamma+\gamma_{1}\left(Y_{t-1}\right)^{\gamma_{2}}$ & $\mathbf{u}_{t} \sim M V N_{2}\left(0, \Sigma_{\mathbf{u}}\right)$ \\
\hline
\end{tabular}

Engle and Manganelli (2004). The usual Quantile Loss (Koenker, 2005) is then used to rank models according their ability to accurately forecast VaR. Namely, letting $\operatorname{VaR}_{t}(\alpha)$ be the $\alpha$-level one-step-ahead VaR forecast at time $t$, the Quantile Loss at level $\alpha\left(Q L_{\alpha}\right)$ is given by

$$
Q L_{\alpha}=\sum_{j=1}^{H}\left(\alpha-L_{T+j}\right)\left(r_{T+j}-\operatorname{VaR}_{T+j}(\alpha)\right), \quad(0<\alpha<1),
$$

where $L_{t}=I\left(r_{t}<\operatorname{VaR}_{t}(\alpha)\right)$.

Finally, to assess the ability of the proposed models to jointly forecast VaR and ES, we rely on the results of Fissler and Ziegel (2016) on the joint elicitability of the couple (VaR, ES). In particular, they show that (VaR, ES) is jointly elicitable with respect to the following class of strictly consistent loss functions

$$
\begin{aligned}
F Z_{t}\left(r_{t}, v_{t}, e_{t} \mid \alpha, G_{1}, G_{2}\right) & =\left(L_{t}-\alpha\right)\left(G_{1}\left(v_{t}\right)-G_{1}\left(r_{t}\right)+\frac{1}{\alpha} G_{2}\left(e_{t}\right) v_{t}\right) \\
& -G_{2}\left(e_{t}\right)\left(\frac{1}{\alpha} L_{t} r_{t}-e_{t}\right)-\mathscr{G}_{2}\left(e_{t}\right),
\end{aligned}
$$

where $G_{1}$ is weakly increasing, $G_{2}$ is strictly increasing and strictly positive, and $\mathscr{G}_{2}^{\prime}=$ $G_{2}$. It can be shown that the expected value of the loss in (39) is uniquely minimized by setting $v_{t}$ and $e_{t}$ equal to the level- $\alpha \mathrm{VaR}$ and ES series, respectively. Following Patton et al. (2019), we assume VaR and ES to be strictly negative and $E S_{t}(\alpha) \leq \operatorname{VaR}_{t}(\alpha)<0$, with $G_{1}(x)=0$ and $G_{2}(x)=-1 / x$, resulting in the following loss function

$$
F Z_{t}^{(0)}=\frac{1}{\alpha E S_{t}(\alpha)} L_{t}\left(r_{t}-\operatorname{VaR}_{t}(\alpha)\right)+\frac{\operatorname{VaR}_{t}(\alpha)}{E S_{t}(\alpha)}+\log \left(-E S_{t}(\alpha)\right)-1,
$$

where $E S_{t}(\alpha)$ is the $\alpha$-level one-step-ahead ES at time $t$. As for the other loss functions, models that, over the chosen forecasting period, show lower average values of $F Z_{t}^{(0)}$ are preferred.

The significance of performance gaps across different models is assessed by means of the Model Confidence Set (MCS) (Hansen et al., 2011). 
Table 7: Predictive Partial log-likelihood $\left(\hat{\ell}_{r}\right)$ and MCS p-values using both Range (pvalue R) and Semi-Quadratic (p-value SQ) statistics. For each market index, we report in bold the highest maximized log-likelihood value, in box models $\in 90 \%$ MCS and in box models $\in 75 \%$ MCS. The out-of-sample period for the market indices is 2008/06/01 - 2018/04/30, for a total of 2492 for DAX 30, 2473 for FTSE 100, 2396 for Hang Seng and 2456 for S\&P 500.

\begin{tabular}{|c|c|c|c|c|c|c|c|c|c|c|c|c|}
\hline & \multicolumn{3}{|c|}{ DAX 30} & \multicolumn{3}{|c|}{ FTSE 100} & \multicolumn{3}{|c|}{ HSI } & \multicolumn{3}{|c|}{ S\&P 500} \\
\hline & $\hat{\ell}_{r}$ & $\mathrm{p}$-value $R$ & $\mathrm{p}$-value $S Q$ & $\hat{\ell}_{r}$ & $\mathrm{p}$-value $\mathrm{R}$ & $\mathrm{p}$-value $S Q$ & $\hat{\ell}_{r}$ & $\mathrm{p}$-value $\mathrm{R}$ & $\mathrm{p}$-value $S Q$ & $\hat{\ell}_{r}$ & $\mathrm{p}$-value $\mathrm{R}$ & $\mathrm{p}$-value $S Q$ \\
\hline RGARCH & 8070.297 & 0.0002 & 0.0044 & 8668.795 & 0.0266 & 0.0674 & 8175.131 & 0.0840 & 0.0394 & 8856.416 & 0.1236 & 0.2352 \\
\hline TV-RGARCH ${ }^{*}$ S2 & 8079.344 & 0.7550 & 0.7062 & 8676.778 & 0.8720 & 0.8566 & 8181.348 & 0.3012 & 0.1780 & 8860.155 & 0.7496 & 0.6716 \\
\hline TV-RGARCH ${ }^{*}$-S & 8079.940 & 0.8546 & 0.7914 & 8677.034 & 0.8720 & 0.8566 & 8182.594 & 0.4852 & 0.3628 & 8860.901 & 0.7960 & 0.8566 \\
\hline TV-RGARCH ${ }^{*}-2$ & 8079.059 & 0.1378 & 0.3638 & 8676.838 & 0.8652 & 0.8566 & 8182.614 & 0.3012 & 0.3134 & 8860.358 & 0.7496 & 0.7164 \\
\hline TV-RGARCH & 8079.773 & 0.8546 & 0.7914 & 8677.070 & 0.8720 & 0.8566 & 8182.023 & 0.1504 & 0.1352 & 8860.609 & 0.7496 & 0.7164 \\
\hline TV-RGARCH & 8079.530 & 0.3694 & 0.6176 & 8676.870 & 0.8652 & 0.8566 & 8182.835 & 0.4852 & 0.3628 & 8861.089 & 0.9914 & 0.9914 \\
\hline ETV-RGARCH & 8080.683 & 1.0000 & 1.0000 & 8677.526 & 1.0000 & 1.0000 & 8183.642 & 1.0000 & 1.0000 & 8861.099 & 1.0000 & 1.0000 \\
\hline
\end{tabular}

Table 8: Average values of QLIKE loss using 5-min $R V$ as volatility proxy and MCS p-values using both Range (p-value R) and Semi-Quadratic (p-value SQ) statistics. For each market index, we report in bold the minimum loss value, in box models $\epsilon$ $90 \%$ MCS and in box models $\in 75 \%$ MCS. The out-of-sample period for the market indices is 2008/06/01 - 2018/04/30, for a total of 2492 for DAX 30, 2473 for FTSE 100, 2396 for Hang Seng and 2456 for S\&P 500.

\begin{tabular}{|c|c|c|c|c|c|c|c|c|c|c|c|c|}
\hline & \multicolumn{3}{|c|}{ DAX 30} & \multicolumn{3}{|c|}{ FTSE 100} & \multicolumn{3}{|c|}{ HSI } & \multicolumn{3}{|c|}{ S\&P 500} \\
\hline & Average & $p$-value $R$ & $\mathrm{p}$-value $S Q$ & Average & $\mathrm{p}$-value $\mathrm{R}$ & $\mathrm{p}$-value SQ & Average & $p$-value $R$ & $\mathrm{p}$-value SQ & Average & $\mathrm{p}$-value $R$ & $\mathrm{p}$-value $S Q$ \\
\hline RGARCH & -8.2741 & 0.0004 & 0.0016 & -9.0421 & 0.0412 & 0.0732 & -8.8664 & 0.0004 & 0.0018 & -9.0741 & 0.0538 & 0.0546 \\
\hline TV-RGARCH ${ }^{*}$-S2 & -8.2780 & 0.1170 & 0.1202 & -9.0462 & 1.0000 & 1.0000 & -8.8797 & 0.0074 & 0.0068 & -9.0771 & 0.1654 & 0.1274 \\
\hline TV-RGARCH*H $-\mathrm{S}$ & -8.2787 & 0.1580 & 0.1490 & -9.0458 & 0.2040 & 0.4238 & -8.8821 & 0.0076 & 0.0068 & -9.0780 & 0.2058 & 0.1554 \\
\hline TV-RGARCH ${ }^{*}-2$ & -8.2786 & 0.1580 & 0.1490 & -9.0455 & 0.2040 & 0.2770 & -8.8825 & 0.0076 & 0.0084 & -9.0765 & 0.1674 & 0.1390 \\
\hline TV-RGARCH ${ }^{*}$ & -8.2785 & 0.1580 & 0.1490 & -9.0461 & 0.8480 & 0.7760 & -8.8812 & 0.0074 & 0.0068 & -9.0777 & 0.1908 & 0.1554 \\
\hline TV-RGARCH & -8.2787 & 0.1580 & 0.1490 & -9.0455 & 0.1886 & 0.2300 & -8.8826 & 0.0076 & 0.0084 & -9.0782 & 0.2058 & 0.1554 \\
\hline ETV-RGARCH & -8.2814 & 1.0000 & 1.0000 & -9.0458 & 0.8480 & 0.7760 & -8.8853 & 1.0000 & 1.0000 & -9.0797 & 1.0000 & 1.0000 \\
\hline
\end{tabular}


Table 9: One-step ahead Value at Risk backtesting at the risk level $\alpha=0.01$. VRate: violation rate i.e. proportion of returns smaller than VaR in the out-of-sample period. $C C$ p-value and $D Q$ p-value: p-values for the Conditional Coverage and Dynamic Quantile test, respectively. Models showing the violation rate closest to the assumed nominal value are indicated in bold. Boxes indicate p-values lower than $5 \%$. The outof-sample period for the market indices is 2008/06/01 - 2018/04/30, for a total of 2492 daily returns for DAX 30, 2473 for FTSE 100, 2396 for Hang Seng and 2456 for S\&P 500 .

\begin{tabular}{|c|c|c|c|c|c|c|c|c|c|c|c|c|}
\hline & \multicolumn{3}{|c|}{ DAX 30} & \multicolumn{3}{|c|}{ FTSE 100} & \multicolumn{3}{|c|}{ HSI } & \multicolumn{3}{|c|}{ S\&P 500} \\
\hline & VRate & CC p-value & DQp-value & VRate & CC p-value & DQ p-value & VRate & CC p-value & DQ p-value & VRate & CC p-value & DQ p-value \\
\hline RGARCH & 0.0132 & 0.1932 & 0.3669 & 0.0125 & 0.3365 & 0.0598 & 0.0134 & 0.1890 & 0.3133 & 0.0159 & 0.0140 & \begin{tabular}{|l|}
0.0156 \\
\end{tabular} \\
\hline TV-RGARCH ${ }^{*}$-S2 & 0.0112 & 0.6046 & 0.6912 & 0.0113 & 0.5006 & \begin{tabular}{|l|}
0.0471 \\
\end{tabular} & 0.0117 & 0.5181 & 0.4681 & 0.0147 & 0.0556 & 0.0664 \\
\hline TV-RGARCH ${ }^{*}-\mathrm{S}$ & 0.0116 & 0.5158 & 0.6352 & 0.0117 & 0.4554 & 0.5392 & 0.0121 & 0.4243 & 0.4360 & 0.0147 & 0.0556 & 0.0702 \\
\hline TV-RGARCH* -2 & 0.0116 & 0.5158 & 0.6339 & 0.0121 & 0.3988 & 0.6166 & 0.0121 & 0.4243 & 0.4335 & 0.0143 & 0.0832 & 0.1100 \\
\hline TV-RGARCH ${ }^{*}$ & 0.0116 & 0.5158 & 0.6327 & 0.0113 & 0.5006 & 0.6129 & 0.0117 & 0.5181 & 0.4698 & 0.0147 & 0.0556 & 0.0690 \\
\hline TV-RGARCH-S & 0.0116 & 0.5158 & 0.6351 & 0.0117 & 0.4554 & 0.5413 & 0.0121 & 0.4243 & 0.4377 & 0.0147 & 0.0556 & 0.0708 \\
\hline ETV-RGARCH & 0.0128 & 0.2596 & 0.5031 & 0.0121 & 0.3988 & 0.4762 & 0.0121 & 0.4243 & 0.4591 & 0.0155 & 0.0228 & 0.0405 \\
\hline
\end{tabular}

For the predictive partial log-likelihood, the results reported in Table 7 show that the ETV-RGARCH is always returning the minimum value of the (negative) predictive partial log-likelihood and both the TV-RGARCH and ETV-RGARCH are always included in the 75\% MCS for both the Range (R) and Semi-Quadratic (SQ) statistics. The standard RGARCH model is always excluded from the MCS at both levels considered for DAX 30, FTSE 100 and HSI and enters the 90\% MCS only for S\&P 500.

Moving to consider the QLIKE loss (Table 8), the ETV-RGARCH is returning the minimum value of the loss function in three cases out of four and is the only model always included in the $75 \%$ MCS for both the R and SQ statistics. For HSI, no other model is included in the MCS at any level while, for the remaining indices and for both $\mathrm{R}$ and SQ, the considered variants of the TV-RGARCH enter the MCS at the $90 \%$ or $75 \%$ level. The RGARCH model is always excluded from the MCS for both confidence levels and test statistics considered.

Next we consider the results of VaR backtesting for two different risk levels: 0.01 and 0.025 . At the 0.01 level (Table 9), models incorporating a correction for dynamic attenuation bias always pass the diagnostic tests at the usual 5\% level. The only exceptions are the ETV-RGARCH model, signficant only at the $1 \%$ level for the S\&P 500, and the TV-RGARCH*-S2, for which, in the case of the FTSE 100, the DQ tests returns a p-value slightly below $5 \%$. The RGARCH model, although performing well for the other three indices, does not pass the diagnostic tests for the S\&P 500. It should however be noted that, for the S\&P 500 dataset, all the models considered have a borderline performance returning p-values very close to the 5\% acceptance threshold. Differently, at the 0.025 level (Table 10), the TV-RGARCH type models are always passing the diagnostic tests while, for the DAX 30, the RGARCH model does not pass the DQ test.

When considering the accuracy in predictive VaR, assessed via the Quantile Loss, we find that, at the 0.01 level (Table 11), the TV-RGARCH is the only model always included in the $75 \%$ MCS for, both the R and SQ statistics, while the ETV-RGARCH 
Table 10: One-step ahead Value at Risk backtesting at the risk level $\alpha=0.025$. VRate: violation rate i.e. proportion of returns smaller than $\mathrm{VaR}$ in the out-of-sample period. $C C$ p-value and DQ p-value: p-values for the Conditional Coverage and Dynamic Quantile test, respectively. Models showing the violation rate closest to the assumed nominal value are indicated in bold. Boxes indicate p-values lower than $5 \%$. The outof-sample period for the market indices is 2008/06/01 - 2018/04/30, for a total of 2492 daily returns for DAX 30, 2473 for FTSE 100, 2396 for Hang Seng and 2456 for S\&P 500 .

\begin{tabular}{|c|c|c|c|c|c|c|c|c|c|c|c|c|}
\hline & \multicolumn{3}{|c|}{ DAX 30} & \multicolumn{3}{|c|}{ FTSE 100} & \multicolumn{3}{|c|}{ HSI } & \multicolumn{3}{|c|}{ S\&P 500} \\
\hline & VRate & CC p-value & DQ p-value & VRate & CC p-value & DQ p-value & VRate & CC p-value & DQ p-value & VRate & CC p-value & DQ p-value \\
\hline RGARCH & 0.0321 & 0.0901 & \begin{tabular}{|l|}
0.0069 \\
\end{tabular} & 0.0295 & 0.1906 & 0.0889 & 0.0263 & 0.5778 & 0.9433 & 0.0293 & 0.4094 & 0.7492 \\
\hline TV-RGARCH ${ }^{*}$-S2 & 0.0289 & 0.4772 & 0.2694 & 0.0275 & 0.2759 & 0.5183 & 0.0275 & 0.2577 & 0.6865 & 0.0281 & 0.6280 & 0.8063 \\
\hline TV-RGARCH ${ }^{*}-\mathrm{S}$ & 0.0293 & 0.4070 & 0.2405 & 0.0263 & 0.6028 & 0.7301 & 0.0275 & 0.2577 & 0.5997 & 0.0281 & 0.6280 & 0.8178 \\
\hline TV-RGARCH ${ }^{*}-2$ & 0.0297 & 0.3418 & 0.2222 & 0.0259 & 0.9287 & 0.8624 & 0.0275 & 0.2577 & 0.6001 & 0.0281 & 0.6280 & 0.8227 \\
\hline TV-RGARCH ${ }^{*}$ & 0.0293 & 0.4070 & 0.2389 & 0.0271 & 0.2819 & 0.5128 & 0.0271 & 0.2627 & 0.7164 & 0.0281 & 0.6280 & 0.8146 \\
\hline TV-RGARCH & 0.0301 & 0.2825 & 0.2583 & 0.0259 & 0.9287 & 0.8626 & 0.0275 & 0.2577 & 0.6045 & 0.0281 & 0.6280 & 0.8198 \\
\hline ETV-RGARCH & 0.0305 & 0.1427 & 0.2238 & 0.0271 & 0.7978 & 0.8649 & 0.0259 & 0.2484 & 0.5682 & 0.0289 & 0.4799 & 0.8336 \\
\hline
\end{tabular}

Table 11: Average Quantile Loss function at the risk level $\alpha=0.01$ and MCS p-values using both Range (p-value R) and Semi-Quadratic (p-value SQ) statistics. For each market index, we report in bold the minimum loss value, in box models $\in 90 \%$ MCS and in box models $\in 75 \%$ MCS. The out-of-sample period for the market indices is 2008/06/01 - 2018/04/30, for a total of 2492 for DAX 30, 2473 for FTSE 100, 2396 for Hang Seng and 2456 for S\&P 500.

\begin{tabular}{|c|c|c|c|c|c|c|c|c|c|c|c|c|}
\hline & \multicolumn{3}{|c|}{ DAX 30} & \multicolumn{3}{|c|}{ FTSE 100} & \multicolumn{3}{|c|}{ HSI } & \multicolumn{3}{|c|}{ S\&P 500} \\
\hline & Average & $p$-value $R$ & $\mathrm{p}$-value SQ & Average & p-value $R$ & $\mathrm{p}$-value $S Q$ & Average & $p$-value $R$ & $\mathrm{p}$-value $S Q$ & Average & $p$-value $R$ & $\mathrm{p}$-value $S Q$ \\
\hline RGARCH & 0.7984 & 0.1058 & 0.2030 & 0.6214 & 0.0004 & 0.0024 & 0.7134 & 0.1168 & 0.2192 & 0.6295 & 0.0014 & 0.0010 \\
\hline TV-RGARCH ${ }^{*}$-S2 & 0.7899 & 0.7684 & 0.6698 & 0.6100 & 0.4922 & 0.4946 & 0.7076 & 0.2470 & 0.3192 & 0.6224 & 0.0014 & 0.0018 \\
\hline TV-RGARCH ${ }^{*}-\mathrm{S}$ & 0.7886 & 1.0000 & 1.0000 & 0.6087 & 1.0000 & 1.0000 & 0.7067 & 0.4184 & 0.3520 & 0.6193 & 0.1732 & 0.2430 \\
\hline TV-RGARCH ${ }^{*}-2$ & 0.7892 & 0.7684 & 0.6698 & 0.6093 & 0.4998 & 0.5628 & 0.7076 & 0.2470 & 0.2932 & 0.6188 & 0.6834 & 0.6834 \\
\hline TV-RGARCH ${ }^{*}$ & 0.7892 & 0.7684 & 0.6698 & 0.6089 & 0.7768 & 0.7768 & 0.7075 & 0.2470 & 0.2932 & 0.6204 & 0.0014 & 0.0046 \\
\hline TV-RGARCH & 0.7889 & 0.7684 & 0.6698 & 0.6093 & 0.4998 & 0.5628 & 0.7062 & 0.4184 & 0.3520 & 0.6186 & 1.0000 & 1.0000 \\
\hline ETV-RGARCH & 0.7950 & 0.6656 & 0.5460 & 0.6119 & 0.4854 & 0.3806 & 0.7038 & 1.0000 & 1.0000 & 0.6229 & 0.0014 & 0.0046 \\
\hline
\end{tabular}

Table 12: Average Quantile Loss function at the risk level $\alpha=0.025$ and MCS pvalues using both Range (p-value R) and Semi-Quadratic (p-value SQ) statistics. For each market index, we report in bold the minimum loss value, in box models $\epsilon$ $90 \%$ MCS and in box models $\in 75 \%$ MCS. The out-of-sample period for the market indices is 2008/06/01 - 2018/04/30, for a total of 2492 for DAX 30, 2473 for FTSE 100, 2396 for Hang Seng and 2456 for S\&P 500.

\begin{tabular}{|c|c|c|c|c|c|c|c|c|c|c|c|c|}
\hline & \multicolumn{3}{|c|}{ DAX 30} & \multicolumn{3}{|c|}{ FTSE 100} & \multicolumn{3}{|c|}{ HSI } & \multicolumn{3}{|c|}{ S\&P 500} \\
\hline & Average & $\mathrm{p}$-value $\mathrm{R}$ & $\mathrm{p}$-value $S Q$ & Average & p-value $R$ & $\mathrm{p}$-value $S Q$ & Average & $\mathrm{p}$-value $\mathrm{R}$ & $\mathrm{p}$-value $S Q$ & Average & $\mathrm{p}$-value $\mathrm{R}$ & $\mathrm{p}$-value $S Q$ \\
\hline RGARCH & 1.7258 & 0.0022 & 0.0350 & 1.3221 & 0.0094 & 0.0140 & 1.4184 & 0.0500 & 0.0430 & 1.3164 & 0.0014 & 0.0002 \\
\hline TV-RGARCH ${ }^{*}$-S2 & 1.7065 & 0.5556 & 0.6260 & 1.3089 & 0.3106 & 0.3950 & 1.4011 & 0.1056 & 0.1022 & 1.2997 & 0.0092 & 0.0166 \\
\hline TV-RGARCH ${ }^{*}-\mathrm{S}$ & 1.7047 & 1.0000 & 1.0000 & 1.3053 & 1.0000 & 1.0000 & 1.3963 & 0.1056 & 0.2172 & 1.2951 & 0.2748 & 0.2908 \\
\hline TV-RGARCH ${ }^{*}-2$ & 1.7052 & 0.8234 & 0.8496 & 1.3059 & 0.7922 & 0.6484 & 1.3963 & 0.1056 & 0.2172 & 1.2932 & 1.0000 & 1.0000 \\
\hline TV-RGARCH ${ }^{*}$ & 1.7049 & 0.8368 & 0.8496 & 1.3061 & 0.7922 & 0.6484 & 1.3981 & 0.1056 & 0.1304 & 1.2966 & 0.0368 & 0.0592 \\
\hline TV-RGARCH & 1.7056 & 0.5556 & 0.6260 & 1.3057 & 0.7922 & 0.6484 & 1.3950 & 0.5488 & 0.5488 & 1.2941 & 0.4624 & 0.4624 \\
\hline ETV-RGARCH & 1.7185 & 0.2594 & 0.2914 & 1.3074 & 0.7922 & 0.6484 & 1.3925 & 1.0000 & 1.0000 & 1.2967 & 0.2748 & 0.2908 \\
\hline
\end{tabular}


Table 13: Average $F Z^{(0)}$ loss function at the risk level $\alpha=0.01$ and MCS p-values using both Range (p-value R) and Semi-Quadratic (p-value SQ) statistics. For each market index, we report in bold the minimum loss value, in box models $\in 90 \%$ MCS and in box models $\in 75 \%$ MCS. The out-of-sample period for the market indices is 2008/06/01 - 2018/04/30, for a total of 2492 for DAX 30, 2473 for FTSE 100, 2396 for Hang Seng and 2456 for S\&P 500.

\begin{tabular}{|c|c|c|c|c|c|c|c|c|c|c|c|c|}
\hline & \multicolumn{3}{|c|}{ DAX 30} & \multicolumn{3}{|c|}{ FTSE 100} & \multicolumn{3}{|c|}{ HSI } & \multicolumn{3}{|c|}{ S\&P 500} \\
\hline & Average & $\mathrm{p}$-value $\mathrm{R}$ & $\mathrm{p}$-value $S Q$ & Average & $p$-value $R$ & $\mathrm{p}$-value $S Q$ & Average & $\mathrm{p}$-value $\mathrm{R}$ & $p$-value $S Q$ & Average & $\mathrm{p}$-value $\mathrm{R}$ & $\mathrm{p}$-value $S Q$ \\
\hline RGARCH & -3.4690 & 0.0002 & 0.0818 & -3.7516 & 0.0008 & 0.0106 & -3.5748 & 0.1208 & 0.1566 & -3.7224 & 0.0124 & 0.0264 \\
\hline TV-RGARCH ${ }^{*}$-S2 & -3.4834 & 0.3670 & 0.4266 & -3.7732 & 0.5256 & 0.5238 & -3.5872 & 0.5370 & 0.5156 & -3.7327 & 0.0124 & 0.0220 \\
\hline TV-RGARCH ${ }^{*}-\mathrm{S}$ & -3.4868 & 0.5458 & 0.5458 & -3.7766 & 1.0000 & 1.0000 & -3.5891 & 0.5370 & 0.5156 & -3.7387 & 0.0880 & 0.1518 \\
\hline TV-RGARCH ${ }^{*}-2$ & -3.4858 & 0.3670 & 0.4514 & -3.7761 & 0.8300 & 0.8234 & -3.5880 & 0.1208 & 0.3898 & -3.7386 & 0.3682 & 0.2574 \\
\hline TV-RGARCH ${ }^{*}$ & -3.4855 & 0.3670 & 0.4514 & -3.7760 & 0.8300 & 0.8234 & -3.5880 & 0.1208 & 0.3898 & -3.7362 & 0.0124 & 0.0264 \\
\hline TV-RGARCH & -3.4864 & 0.5310 & 0.5306 & -3.7756 & 0.5562 & 0.6646 & -3.5897 & 0.5370 & 0.5156 & -3.7407 & 0.3682 & 0.2574 \\
\hline ETV-RGARCH & -3.4918 & 1.0000 & 1.0000 & -3.7751 & 0.8300 & 0.8234 & -3.5934 & 1.0000 & 1.0000 & -3.7475 & 1.0000 & 1.0000 \\
\hline
\end{tabular}

Table 14: Average $F Z^{(0)}$ loss function at the risk level $\alpha=0.025$ and MCS p-values using both Range (p-value R) and Semi-Quadratic (p-value SQ) statistics. For each market index, we report in bold the minimum loss value, in box models $\in 90 \%$ MCS and in box models $\in 75 \%$ MCS. The out-of-sample period for the market indices is 2008/06/01 - 2018/04/30, for a total of 2492 for DAX 30, 2473 for FTSE 100, 2396 for Hang Seng and 2456 for S\&P 500.

\begin{tabular}{|c|c|c|c|c|c|c|c|c|c|c|c|c|}
\hline & \multicolumn{3}{|c|}{ DAX 30} & \multicolumn{3}{|c|}{ FTSE 100} & \multicolumn{3}{|c|}{ HSI } & \multicolumn{3}{|c|}{ S\&P 500} \\
\hline & Average & $p$-value $R$ & $\mathrm{p}$-value $S Q$ & Average & $p$-value $R$ & $\mathrm{p}$-value SQ & Average & $p$-value $R$ & $\mathrm{p}$-value $S Q$ & Average & $\mathrm{p}$-value $\mathrm{R}$ & $\mathrm{p}$-value $S Q$ \\
\hline RGARCH & -3.6346 & 0.0128 & 0.0850 & -3.9302 & 0.0180 & 0.0232 & -3.7916 & 0.0254 & 0.0304 & -3.9322 & 0.0026 & 0.0016 \\
\hline TV-RGARCH ${ }^{*}$-S2 & -3.6507 & 0.7078 & 0.7698 & -3.9409 & 0.3416 & 0.3202 & -3.8077 & 0.1280 & 0.1488 & -3.9462 & 0.0098 & 0.0024 \\
\hline TV-RGARCH* ${ }^{*}$ - & -3.6527 & 1.0000 & 1.0000 & -3.9456 & 0.8370 & 0.8370 & -3.8108 & 0.3742 & 0.2840 & -3.9512 & 0.0550 & 0.0624 \\
\hline TV-RGARCH ${ }^{*}-2$ & -3.6521 & 0.7078 & 0.8564 & -3.9452 & 0.7824 & 0.8056 & -3.8106 & 0.1280 & 0.1822 & -3.9526 & 0.2100 & 0.2172 \\
\hline TV-RGARCH ${ }^{*}$ & -3.6522 & 0.7078 & 0.8564 & -3.9443 & 0.6018 & 0.6874 & -3.8098 & 0.1280 & 0.1822 & -3.9495 & 0.0098 & 0.0066 \\
\hline TV-RGARCH & -3.6521 & 0.7078 & 0.8564 & -3.9451 & 0.7824 & 0.8056 & -3.8115 & 0.3742 & 0.2840 & -3.9524 & 0.2100 & 0.2172 \\
\hline ETV-RGARCH & -3.6515 & 0.7078 & 0.8564 & -3.9460 & 1.0000 & 1.0000 & -3.8143 & 1.0000 & 1.0000 & -3.9581 & 1.0000 & 1.0000 \\
\hline
\end{tabular}

models enters the 75\% MCS for all indices except for the S\&P 500. The RGARCH is always excluded from the MCS for FTSE 100 and S\&P 500 but it enters the 90\% MCS for DAX 30 and HSI. For the 0.025 level (Table 12), we find that the TV-RGARCH and ETV-RGARCH are the only models always included in the $75 \%$ MCS while, on the other hand, the RGARCH never enters the MCS.

A similar picture is observed when jointly evaluating the quality of VaR and ES forecasts via the $F Z^{(0)}$ loss function (Tables 13 and 14). For both the 0.01 and 0.025 levels the following facts arise: both the TV-RGARCH and ETV-RGARCH models are always entering the $75 \% \mathrm{MCS}$, with one exception: the TV-RGARCH for S\&P 500 at the 0.025 risk level only enters the $90 \%$ MCS; the RGARCH-model is never included in the $75 \%$ MCS and enters the 90\% MCS only for the HSI at the 0.01 level; For both risk levels, the ETV-RGARCH model is returning the minimum average $F Z^{(0)}$ value for three out of the four indices considered (excluding FTSE 100, for the 0.01 level, and DAX 30, for the 0.025 risk level).

In conclusion: the results of our out-of-sample forecasting experiment show that: i) for both volatility and tail risk forecasting, the proposed time-varying 
RGARCH models, TV-RGARCH and ETV-RGARCH, always outperform the standard RGARCH model; ii) the proposed TV-RGARCH and ETV-RGARCH models are not outperformed by the alternative time-varying specifications considered as robustness checks; iii) the results are in general robust to the specification of the functional form of the $\gamma_{t}$ and $\beta_{t}$ coefficients.

\section{Concluding Remarks}

We have proposed novel model specifications that generalize the log-linear RGARCH model by Hansen et al. (2012), so as to account for time-varying attenuation bias effects. The proposed models appear to be effective in capturing the dependency of volatility dynamics on the variability of the measurement error of the reference logtransformed realized measure. The results of an application to VaR and ES forecasting, for four major stock market indices, support the profitability of the proposed model in risk management applications. Estimation of model parameters can be efficiently done via ML estimation. Furthermore, accurate finite sample inference has been obtained implementing a parametric Bootstrap procedure.

The derivation of the statistical properties of the proposed models is an interesting but challenging issue that has not been investigated in this paper. Although it is easy to show that, as for the standard RGARCH model, the TV-RGARCH model, and its extensions, can be written as Hidden Markov Models, depending on a latent Markov chain, analytical derivation of stationarity and ergodicity conditions is made troublesome by the non-linearity of the latent chain; investigation of these issues has been currently left for future research.

\section{References}

Andersen, T. G., T. Bollerslev, F. X. Diebold, and P. Labys (2001). The distribution of realized exchange rate volatility. Journal of the American statistical association 96(453), 42-55.

Barndorff-Nielsen, O. E. and N. Shephard (2002). Econometric analysis of realized volatility and its use in estimating stochastic volatility models. Journal of the Royal Statistical Society: Series B (Statistical Methodology) 64(2), 253-280.

Bollerslev, T. (1988). On the correlation structure for the generalized autoregressive conditional heteroskedastic process. Journal of Time Series Analysis 9(2), 121131.

Bollerslev, T., A. J. Patton, and R. Quaedvlieg (2016). Exploiting the errors: A simple approach for improved volatility forecasting. Journal of Econometrics 192(1), 1 -18 .

Borup, D. and J. S. Jakobsen (2019). Capturing volatility persistence: a dynamically complete realized egarch-midas model. Quantitative Finance 19(11), 1839-1855. 
Buccheri, G. and F. Corsi (2019). HARK the SHARK: Realized Volatility Modeling with Measurement Errors and Nonlinear Dependencies. Journal of Financial Econometrics. forthcoming.

Christoffersen, P. F. (1998). Evaluating interval forecasts. International Economic Review 39(4), 841-862.

Corsi, F. (2009). A simple approximate long-memory model of realized volatility. Journal of Financial Econometrics, 174-196.

Corsi, F., S. Mittnik, C. Pigorsch, and U. Pigorsch (2008). The volatility of realized volatility. Econometric Reviews 27(1-3), 46-78.

Engle, R. (2002). New frontiers for arch models. Journal of Applied Econometrics 17(5), 425-446.

Engle, R. F. and G. M. Gallo (2006). A multiple indicators model for volatility using intra-daily data. Journal of Econometrics 131(1), 3-27.

Engle, R. F. and S. Manganelli (2004). Caviar: Conditional autoregressive value at risk by regression quantiles. Journal of Business \& Economic Statistics 22(4), 367-381.

Fissler, T. and J. F. Ziegel (2016). Higher order elicitability and Osband's principle. The Annals of Statistics 44(4), 1680-1707.

Hansen, P. and A. Lunde (2011). Forecasting volatility using high frequency data. The Oxford Handbook of Economic Forecasting, Oxford: Blackwell, 525-556.

Hansen, P. R. and Z. Huang (2016). Exponential garch modeling with realized measures of volatility. Journal of Business \& Economic Statistics 34(2), 269287.

Hansen, P. R., Z. Huang, and H. H. Shek (2012). Realized garch: a joint model for returns and realized measures of volatility. Journal of Applied Econometrics 27(6), 877-906.

Hansen, P. R., A. Lunde, and J. M. Nason (2011). The model confidence set. Econometrica 79(2), 453-497.

Koenker, R. (2005). Quantile Regression. Econometric Society Monographs. Cambridge University Press.

Kristensen, D. and O. Linton (2006). A closed-form estimator for the garch(1,1) model. Econometric Theory 22(2), 323-337.

Li, Y.-N., Y. Zhang, and C. Zhang (2019). Statistical inference for measurement equation selection in the log-realgarch model. Econometric Theory 35(5), 943977. 
Liu, L. Y., A. J. Patton, and K. Sheppard (2015). Does anything beat 5-minute rv? a comparison of realized measures across multiple asset classes. Journal of Econometrics 187(1), 293-311.

Paparoditis, E. and D. Politis (2009). Handbook of Financial Time Series, Chapter Resampling and Subsampling for Financial Time Series. Springer.

Patton, A. (2011). Volatility forecast comparison using imperfect volatility proxies. Journal of Econometrics 160(1), 246-256.

Patton, A., D. N. Politis, and H. White (2009). Correction to "automatic block-length selection for the dependent bootstrap" by d. politis and h. white. Econometric Reviews 28(4), 372-375.

Patton, A. J., J. F. Ziegel, and R. Chen (2019). Dynamic semiparametric models for expected shortfall (and value-at-risk). Journal of Econometrics 211(2), 388 - 413.

Shephard, N. and K. Sheppard (2010). Realising the future: forecasting with high-frequency-based volatility (heavy) models. Journal of Applied Econometrics 25(2), 197-231.

Shephard, N. and D. Xiu (2016). Econometric analysis of multivariate realised qml: efficient positive semi-definite estimators of the covariation of equity prices. 


\section{Empirical Appendix}

Table 15: Summary statistics

\begin{tabular}{|c|c|c|c|c|c|c|c|c|}
\hline & \multicolumn{2}{|c|}{ DAX 30} & \multicolumn{2}{|c|}{ FTSE 100} & \multicolumn{2}{|c|}{ HSI } & \multicolumn{2}{|c|}{ S\&P 500} \\
\hline & $r_{t}$ & $R V_{t}^{\dagger}$ & $r_{t}$ & $R V_{t}^{\dagger}$ & $r_{t}$ & $R V_{t}^{\dagger}$ & $r_{t}$ & $R V_{t}^{\dagger}$ \\
\hline n.obs & 4096 & 4096 & 4063 & 4063 & 3951 & 3951 & 4014 & 4014 \\
\hline Min & -0.071 & 0.035 & -0.070 & 0.026 & -0.132 & 0.039 & -0.082 & 0.017 \\
\hline Max & 0.092 & 67.627 & 0.088 & 58.520 & 0.095 & 40.069 & 0.074 & 57.833 \\
\hline Q1 & -0.005 & 0.396 & -0.004 & 0.172 & -0.005 & 0.222 & -0.004 & 0.181 \\
\hline Median & 0.001 & 0.749 & 0.000 & 0.305 & 0.000 & 0.368 & 0.000 & 0.334 \\
\hline Q3 & 0.006 & 1.512 & 0.005 & 0.643 & 0.004 & 0.628 & 0.004 & 0.736 \\
\hline Mean & 0.000 & 1.525 & 0.000 & 0.677 & -0.001 & 0.661 & 0.000 & 0.814 \\
\hline Stdev & 0.012 & 2.762 & 0.009 & 1.661 & 0.010 & 1.425 & 0.010 & 2.041 \\
\hline Skew & 0.127 & 8.303 & 0.096 & 16.169 & -0.220 & 13.531 & -0.204 & 11.571 \\
\hline Kurt & 5.216 & 123.868 & 8.403 & 430.361 & 15.997 & 275.734 & 9.306 & 214.716 \\
\hline
\end{tabular}

Summary statistics of daily log-returns $r_{t}$ and daily Realized Volatilities $R V_{t} \quad(\dagger$ : intra-daily returns $r_{t, i} \times 100$ ) for the stock market indices DAX 30, FTSE 100, Hang Seng and S\&P500 for the full sample period 2002/01/01 - 2018/04/30. n.obs: number of observations for each series; Min: Minimum; Max: Maximum; Q1: First Quartile; Q3: Third Quartile; Median; Mean; Stdev: Standard deviation; Skew: Skewness; Kurt: Kurtosis. 
Figure 7: Histograms and superimposed non-parametric densities of 999 standardized Bootstrap estimates of the parameters of the RGARCH model
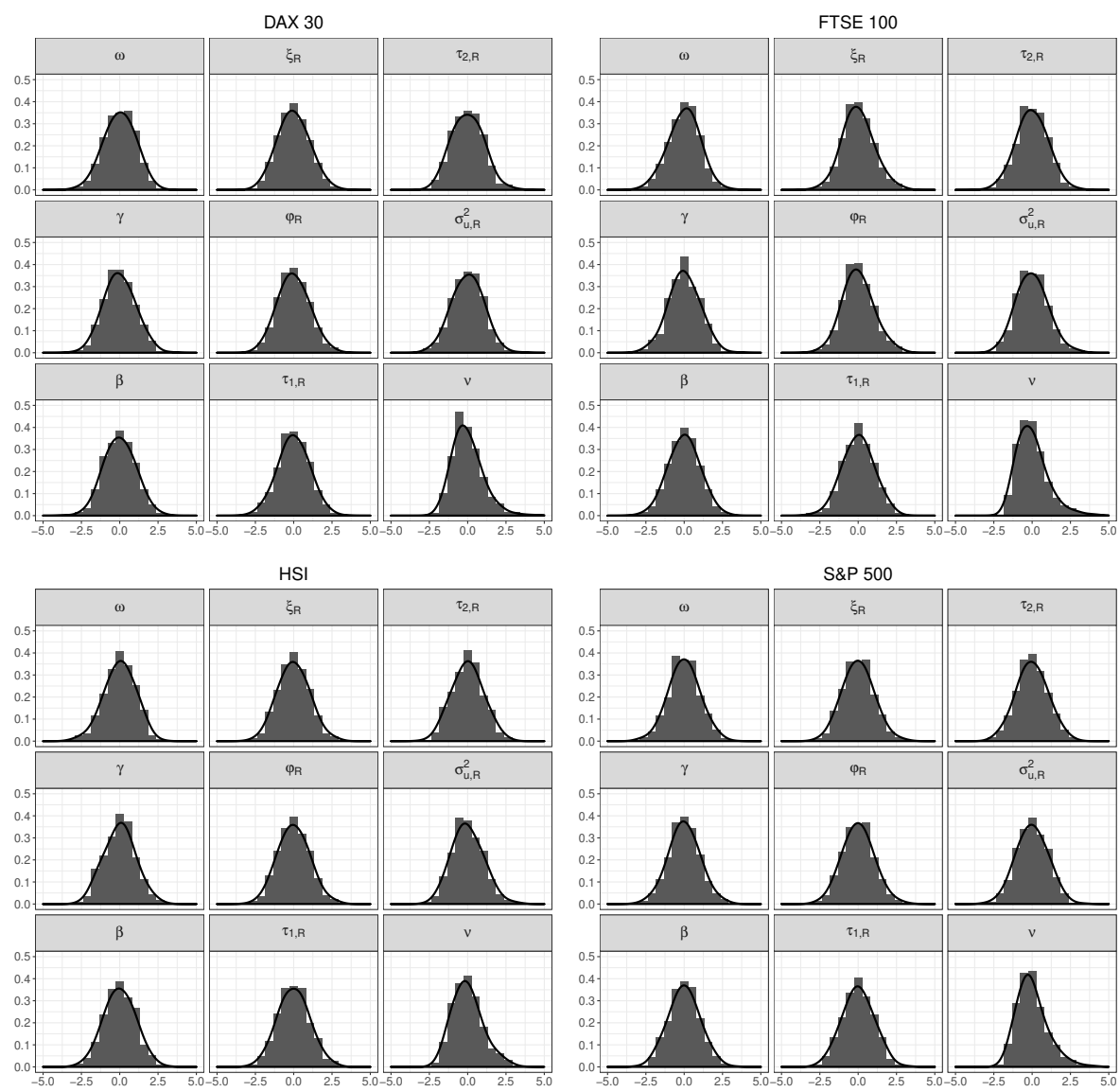
Figure 8: Histograms and superimposed non-parametric densities of 999 standardized Bootstrap estimates of the parameters of the TV-RGARCH model
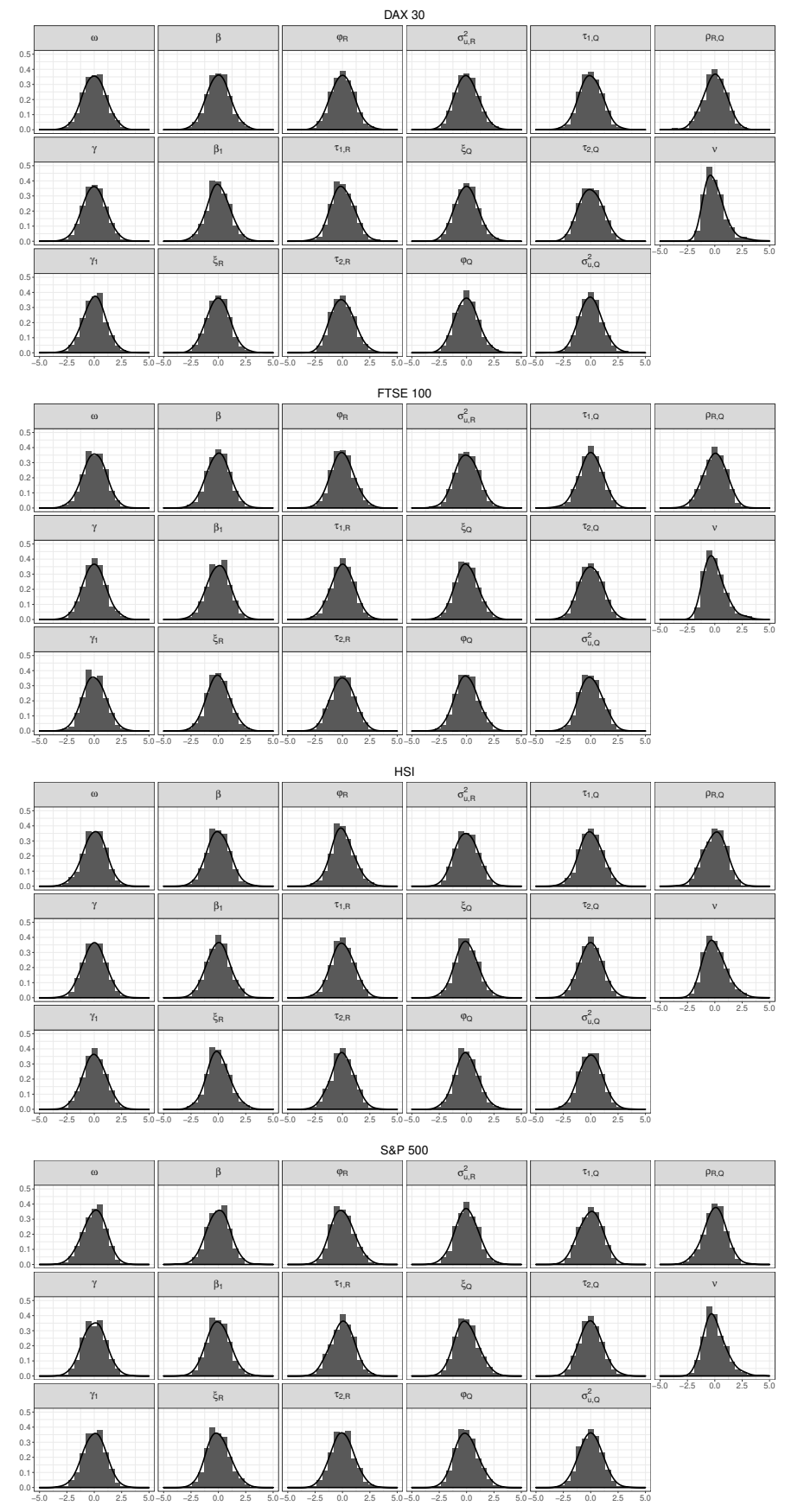
Table 16: Summary of Bootstrap estimates for the RGARCH model

\begin{tabular}{|c|c|c|c|c|c|c|c|c|c|c|c|c|c|c|c|c|c|c|c|c|c|c|c|c|}
\hline & \multicolumn{6}{|c|}{ DAX 30} & \multicolumn{6}{|c|}{ FTSE 100} & \multicolumn{6}{|c|}{ HSI } & \multicolumn{6}{|c|}{ S\&P 500 } \\
\hline & Coef & $\mu_{B}$ & SE & SE.B & $q_{0.025}$ & $q_{0.975}$ & Coef & $\mu_{B}$ & SE & SE.B & $q_{0.025}$ & $q_{0.975}$ & Coef & $\mu_{B}$ & SE & SE.B & $q_{0.025}$ & & Coef & $\mu_{B}$ & SE & SE.B & $q_{0.025}$ & $q_{0.975}$ \\
\hline$\omega$ & -0.171 & -0.178 & 0.082 & 0.112 & -0.393 & 0.029 & -0.039 & -0.046 & 0.089 & 0.110 & -0.267 & 0.163 & -0.096 & -0.106 & 0.062 & 0.089 & -0.296 & 0.050 & -0.002 & -0.014 & 0.109 & 0.123 & -0.252 & 0.229 \\
\hline$\gamma$ & 0.362 & 0.362 & 0.016 & 0.017 & 0.331 & 0.394 & 0.363 & 0.363 & 0.016 & 0.016 & 0.330 & 0.392 & 0.226 & 0.226 & 0.013 & 0.013 & 0.202 & 0.253 & 0.479 & 0.478 & 0.019 & 0.018 & 0.443 & 0.514 \\
\hline$\beta$ & 0.614 & 0.614 & 0.015 & 0.013 & 0.590 & 0.640 & 0.619 & 0.618 & 0.015 & 0.013 & 0.593 & 0.642 & 0.752 & 0.752 & 0.014 & 0.012 & 0.729 & 0.774 & 0.508 & 0.508 & 0.017 & 0.014 & 0.481 & 0.536 \\
\hline$\xi_{R}$ & -0.068 & -0.064 & 0.213 & 0.284 & -0.589 & 0.510 & -0.401 & -0.401 & 0.225 & 0.278 & -0.921 & 0.169 & -0.257 & -0.239 & 0.243 & 0.356 & -0.896 & 0.505 & -0.629 & -0.614 & 0.205 & 0.228 & -1.031 & -0.152 \\
\hline$\varphi_{R}$ & 1.008 & 1.008 & 0.023 & 0.030 & 0.952 & 1.070 & 1.000 & 1.000 & 0.023 & 0.028 & 0.947 & & 23 & 1.025 & 0.025 & 0.037 & 0.957 & 100 & 0.963 & 0.965 & 0.021 & 0.023 & 0.922 & 1.013 \\
\hline$\tau_{1, R}$ & -0.135 & -0.135 & 0.008 & 0.008 & -0.152 & -0.119 & -0.087 & -0.088 & 0.007 & 0.007 & -0.101 & -0.07 & 0.002 & 0.002 & 0.007 & 0.008 & -0.013 & 0.017 & -0.107 & -0.107 & 0.008 & 0.008 & -0.122 & -0.092 \\
\hline$\tau_{2, R}$ & 0.107 & 0.109 & 0.005 & 0.008 & 0.095 & 0.124 & 0.100 & 0.100 & 0.005 & 0.006 & 0.088 & 0.112 & 0.141 & 0.142 & 0.006 & 0.006 & 0.131 & 0.153 & 0.099 & 0.099 & 0.005 & 0.006 & 0.088 & 0.111 \\
\hline$\sigma_{u, R}^{2}$ & 0.219 & 0.219 & 0.005 & 0.006 & 0.207 & 0.231 & 0.181 & 0.180 & 0.004 & 0.006 & 0.170 & 0.192 & 0.212 & 0.211 & 0.005 & 0.007 & 0.199 & 0.225 & 0.234 & 0.234 & 0.005 & 0.006 & 0.222 & 0.246 \\
\hline & 8.696 & 8.923 & 0.811 & 1.347 & 6.797 & 12.012 & 11.565 & 11.944 & 0.520 & 2.084 & 8.913 & 17.064 & 7.049 & 7.169 & 0.342 & 0.731 & 5.976 & 8.778 & 8.493 & 8.704 & 0.258 & 1.200 & 6.840 & 11.553 \\
\hline
\end{tabular}

Coef: estimated coefficient; $\mu_{B}$ : mean of Bootstrap estimates; SE: asymptotic standard error ; SE.B: standard error of Bootstrap estimates; $q_{0.025}: 2.5 \%$ Bootstrap percentile; $q_{0.975}: 97.5 \%$ Bootstrap percentile. 
Table 17: Summary of Bootstrap estimates for the TV-RGARCH model

\begin{tabular}{|c|c|c|c|c|c|c|c|c|c|c|c|c|c|c|c|c|c|c|c|c|c|c|c|c|}
\hline & \multicolumn{6}{|c|}{ DAX 30} & \multicolumn{6}{|c|}{ FTSE 100} & \multicolumn{6}{|c|}{ HSI } & \multicolumn{6}{|c|}{ S\&P 500} \\
\hline & Coef & $\mu_{B}$ & SE & SE.B & $q_{0.025}$ & $q_{0.975}$ & Coef & $\mu_{B}$ & SE & SE.B & $q_{0.025}$ & $q_{0.975}$ & Coef & $\mu_{B}$ & SE & SE.B & $q_{0.025}$ & $q_{0.975}$ & Coef & $\mu_{B}$ & SE & SE.B & $q_{0.025}$ & $q_{0.975}$ \\
\hline$\omega$ & -0.352 & -0.351 & 0.096 & 0.137 & -0.622 & -0.086 & -0.090 & -0.093 & 0.100 & 0.117 & -0.322 & 0.129 & -0.262 & -0.276 & 0.076 & 0.102 & -0.489 & -0.084 & -0.157 & -0.166 & 0.111 & 0.131 & -0.426 & 0.068 \\
\hline$\gamma$ & 0.471 & 0.470 & 0.022 & 0.024 & 0.424 & 0.519 & 0.442 & 0.443 & 0.022 & 0.021 & 0.403 & 0.486 & 0.304 & 0.302 & 0.018 & 0.019 & 0.267 & 0.338 & 0.546 & 0.544 & 0.021 & 0.022 & 0.502 & 0.586 \\
\hline$\gamma_{1}$ & -0.134 & -0.132 & 0.034 & 0.037 & -0.205 & -0.061 & -0.113 & -0.113 & 0.043 & 0.042 & -0.193 & -0.030 & -0.126 & -0.125 & 0.035 & 0.037 & -0.197 & -0.056 & -0.214 & -0.215 & 0.057 & 0.060 & -0.334 & -0.106 \\
\hline$\beta$ & 0.474 & 0.474 & 0.022 & 0.020 & 0.435 & 0.516 & 0.523 & 0.521 & 0.021 & 0.018 & 0.486 & 0.556 & 0.646 & 0.647 & 0.019 & 0.017 & 0.613 & 0.681 & 0.419 & 0.419 & 0.020 & 0.019 & 0.382 & 0.455 \\
\hline$\beta_{1}$ & 0.166 & 0.165 & 0.034 & 0.037 & 0.092 & 0.238 & 0.148 & 0.149 & 0.044 & 0.043 & 0.065 & 0.228 & 0.159 & 0.157 & 0.036 & 0.038 & 0.084 & 0.233 & 0.250 & 0.251 & 0.058 & 0.060 & 0.140 & 0.370 \\
\hline$\xi_{R}$ & 0.008 & 0.004 & 0.210 & 0.295 & -0.561 & 0.592 & -0.385 & -0.394 & 0.221 & 0.253 & -0.876 & 0.121 & -0.380 & -0.348 & 0.251 & 0.319 & -0.980 & 0.329 & -0.557 & -0.555 & 0.188 & 0.226 & -0.981 & -0.082 \\
\hline$\varphi_{R}$ & 1.015 & 1.015 & 0.023 & 0.032 & 0.955 & 1.077 & 1.001 & 1.000 & 0.022 & 0.026 & 0.951 & 1.053 & 1.010 & 1.013 & 0.026 & 0.033 & 0.947 & 1.082 & 0.970 & 0.970 & 0.019 & 0.023 & 0.928 & 1.017 \\
\hline$\tau_{1, R}$ & -0.133 & -0.134 & 0.007 & 0.008 & -0.148 & -0.118 & -0.089 & -0.090 & 0.007 & 0.007 & -0.104 & -0.076 & 0.003 & 0.003 & 0.007 & 0.008 & -0.013 & 0.018 & -0.107 & -0.108 & 0.008 & 0.008 & -0.123 & -0.092 \\
\hline$\tau_{2, R}$ & 0.108 & 0.109 & 0.005 & 0.007 & 0.097 & 0.123 & 0.098 & 0.098 & 0.005 & 0.006 & 0.087 & 0.110 & 0.140 & 0.140 & 0.006 & 0.006 & 0.129 & 0.150 & 0.099 & 0.099 & 0.005 & 0.006 & 0.088 & 0.111 \\
\hline$\sigma_{u, R}^{2}$ & 0.213 & 0.212 & 0.005 & 0.006 & 0.202 & 0.223 & 0.177 & 0.176 & 0.004 & 0.005 & 0.166 & 0.187 & 0.208 & 0.208 & 0.005 & 0.007 & 0.195 & 0.221 & 0.231 & 0.231 & 0.005 & 0.006 & 0.219 & 0.242 \\
\hline$\xi_{Q}$ & -0.015 & -0.014 & 0.208 & 0.285 & -0.556 & 0.563 & -0.289 & -0.297 & 0.221 & 0.251 & -0.775 & 0.195 & -0.817 & -0.785 & 0.249 & 0.298 & -1.351 & -0.150 & -0.767 & -0.766 & 0.182 & 0.223 & -1.165 & -0.311 \\
\hline$\varphi_{Q}$ & 0.979 & 0.979 & 0.022 & 0.030 & 0.922 & 1.040 & 0.984 & 0.984 & 0.022 & 0.025 & 0.935 & & 0.936 & 0.939 & 0.026 & 0.031 & 0.879 & 1.002 & 0.933 & 0.933 & 0.018 & 0.022 & 0.893 & 0.978 \\
\hline$\tau_{1, Q}$ & -0.115 & -0.115 & 0.010 & 0.012 & -0.138 & -0.092 & -0.077 & -0.077 & 0.008 & 0.009 & -0.095 & -0.059 & 0.006 & 0.006 & 0.009 & 0.010 & -0.015 & 0.026 & -0.080 & -0.081 & 0.009 & 0.010 & -0.100 & -0.062 \\
\hline$\tau_{2, Q}$ & 0.137 & 0.140 & 0.007 & 0.011 & 0.119 & 0.161 & 0.114 & 0.115 & 0.006 & 0.008 & 0.101 & 0.130 & 0.153 & 0.153 & 0.007 & 0.007 & 0.140 & 0.166 & 0.110 & 0.110 & 0.006 & 0.008 & 0.096 & 0.126 \\
\hline$\sigma_{u, Q}^{2}$ & 0.366 & 0.365 & 0.008 & 0.010 & 0.346 & 0.385 & 0.273 & 0.273 & 0.006 & 0.009 & 0.255 & 0.290 & 0.326 & 0.326 & 0.007 & 0.010 & 0.306 & 0.346 & 0.291 & 0.291 & 0.007 & 0.008 & 0.276 & 0.306 \\
\hline$\rho$ & 0.911 & 0.911 & 0.003 & 0.003 & 0.905 & 0.917 & 0.927 & 0.927 & 0.002 & 0.003 & 0.922 & 0.932 & 0.932 & 0.932 & 0.002 & 0.002 & 0.927 & 0.937 & 0.946 & 0.946 & 0.002 & 0.002 & 0.942 & 0.950 \\
\hline$v$ & 8.947 & 9.166 & 0.377 & 1.478 & 7.119 & 12.989 & 11.882 & 12.268 & 0.278 & 2.206 & 9.055 & 17.743 & 6.998 & 7.277 & 0.351 & 0.771 & 5.987 & 9.053 & 8.819 & 9.027 & 0.362 & 1.153 & 7.175 & 11.751 \\
\hline
\end{tabular}

Coef: estimated coefficient; $\mu_{B}$ : mean of Bootstrap estimates; SE: asymptotic standard error ; SE.B: standard error of Bootstrap estimates; $q_{0.025}: 2.5 \%$ Bootstrap percentile; $q_{0.975}: 97.5 \%$ Bootstrap percentile. 
Table 18: In-sample estimation results for TV-RGARCH and ETV-RGARCH with time-varying variance of the measurement error $u_{t, R}$.

\begin{tabular}{|c|c|c|c|c|c|c|c|c|c|c|c|c|c|c|c|c|}
\hline \multirow{4}{*}{$\omega$} & \multicolumn{4}{|c|}{ DAX 30} & \multicolumn{4}{|c|}{ FTSE 100} & \multicolumn{4}{|c|}{ HSI } & \multicolumn{4}{|c|}{ S\&P 500 } \\
\hline & TV-HRG & TV-HRG ${ }^{*}$ & ETV-HRG & ETV-HRG ${ }^{*}$ & TV-HRG & TV-HRG & ETV-HRG & ETV-HRG ${ }^{*}$ & TV-HRG & TV-HRG & ETV-HRG & ETV-HRG ${ }^{*}$ & TV-HRG & TV-HRG ${ }^{*}$ & ETV-HRG & ETV-HRG* \\
\hline & -0.345 & -0.345 & $\begin{array}{l}-0.078 \\
\end{array}$ & -0.090 & -0.094 & \begin{tabular}{|c|}
-0.093 \\
\end{tabular} & 0.149 & 0.155 & -0.259 & -0.257 & 0.271 & 0.272 & \begin{tabular}{|c|}
-0.157 \\
\end{tabular} & $\begin{array}{l}-0.157 \\
\end{array}$ & 1.083 & 1.083 \\
\hline & $(0.070)$ & $(0.095)$ & $(0.435)$ & $(0.342)$ & $(0.102)$ & $(0.093)$ & $(0.409)$ & $(0.386)$ & $(0.065)$ & $(0.066)$ & $(0.571)$ & (0.687) & (0.127) & $(0.105)$ & $(0.329)$ & (0.311) \\
\hline \multirow[t]{2}{*}{$\gamma$} & 0.472 & 0.472 & 1.075 & 1.073 & 0.442 & 0.442 & 0.853 & 0.856 & 0.301 & 0.301 & 0.675 & 0.675 & 0.545 & 0.545 & 0.797 & 0.797 \\
\hline & $(0.020)$ & $(0.022)$ & $(0.113)$ & $(0.110)$ & $(0.022)$ & $(0.022)$ & $(0.119)$ & $(0.118)$ & $(0.018)$ & $(0.018)$ & $(0.095)$ & $(0.101)$ & $(0.022)$ & $(0.021)$ & (0.128) & $(0.120)$ \\
\hline \multirow[t]{2}{*}{$\gamma_{1}$} & -0.136 & -0.136 & -0.199 & -0.199 & -0.113 & -0.113 & -0.201 & -0.201 & -0.119 & -0.120 & -0.157 & -0.158 & -0.212 & -0.211 & -0.208 & -0.207 \\
\hline & $(0.035)$ & $(0.035)$ & $(0.038)$ & $(0.037)$ & $(0.041)$ & $(0.042)$ & $(0.049)$ & $(0.049)$ & $(0.034)$ & $(0.034)$ & $(0.035)$ & $(0.035)$ & $(0.058)$ & $(0.058)$ & $(0.060)$ & $(0.059)$ \\
\hline$\gamma_{2}$ & - & - & $\begin{array}{c}0.257 \\
(0.044)\end{array}$ & $\begin{array}{c}0.257 \\
(0.043)\end{array}$ & - & - & $\begin{array}{c}0.237 \\
(0.055)\end{array}$ & $\begin{array}{c}0.238 \\
(0.056)\end{array}$ & - & - & $\begin{array}{c}0.189 \\
(0.039)\end{array}$ & $\begin{array}{c}0.189 \\
(0.039)\end{array}$ & - & - & $\begin{array}{c}0.220 \\
(0.064)\end{array}$ & $\begin{array}{c}0.219 \\
(0.063)\end{array}$ \\
\hline \multirow{2}{*}{$\beta$} & 0.473 & 0.473 & $\begin{array}{l}(0.044) \\
-0.074\end{array}$ & $\begin{array}{l}(0.043) \\
-\mathbf{- 0 . 0 7 4}\end{array}$ & 0.523 & 0.522 & 0.155 & 0.153 & 0.650 & 0.650 & 0.374 & $\begin{array}{c}(0.039) \\
0.374\end{array}$ & 0.419 & 0.419 & $\begin{array}{c}(0.064) \\
0.419\end{array}$ & $\begin{array}{c}(0.063) \\
0.419\end{array}$ \\
\hline & $(0.022)$ & $(0.022)$ & $(0.142)$ & $(0.132)$ & $(0.021)$ & $(0.021)$ & $(0.142)$ & $(0.146)$ & $(0.019)$ & $(0.019)$ & $(0.142)$ & $(0.146)$ & $(0.020)$ & $(0.020)$ & $(0.130)$ & (0.126) \\
\hline \multirow[t]{2}{*}{$\beta_{1}$} & 0.168 & 0.169 & 0.231 & 0.231 & 0.149 & 0.149 & 0.238 & 0.239 & 0.152 & 0.152 & 0.191 & 0.191 & 0.248 & 0.247 & 0.245 & 0.244 \\
\hline & $(0.034)$ & $(0.035)$ & $(0.037)$ & $(0.037)$ & $(0.042)$ & (0.043) & $(0.050)$ & $(0.050)$ & $(0.035)$ & $(0.035)$ & $(0.036)$ & $(0.037)$ & $(0.059)$ & $(0.059)$ & $(0.061)$ & $(0.060)$ \\
\hline \multirow[t]{2}{*}{$\beta_{2}$} & - & - & -0.287 & -0.287 & - & - & -0.273 & -0.273 & - & - & -0.218 & -0.219 & - & - & -0.245 & -0.244 \\
\hline & & & $(0.045)$ & (0.043) & & & $(0.056)$ & $(0.057)$ & & & $(0.041)$ & $(0.041)$ & & & $(0.065)$ & (0.064) \\
\hline \multirow[t]{2}{*}{$\xi_{R}$} & 0.000 & -0.002 & 0.025 & 0.025 & -0.389 & -0.391 & -0.281 & -0.282 & -0.381 & -0.386 & -0.392 & -0.396 & -0.558 & -0.559 & -0.628 & -0.628 \\
\hline & $(0.134)$ & $(0.208)$ & $(0.240)$ & $(0.186)$ & $(0.229)$ & $(0.204)$ & $(0.253)$ & $(0.230)$ & $(0.197)$ & $(0.200)$ & $(0.260)$ & $(0.329)$ & $(0.221)$ & $(0.160)$ & $(0.214)$ & $(0.182)$ \\
\hline \multirow[t]{2}{*}{$\varphi_{R}$} & 1.014 & 1.014 & 1.017 & 1.017 & 1.001 & 1.001 & 1.011 & 1.011 & 1.010 & 1.009 & 1.008 & 1.008 & 0.970 & 0.970 & 0.963 & 0.963 \\
\hline & $(0.016)$ & $(0.022)$ & $(0.026)$ & $(0.020)$ & $(0.023)$ & $(0.021)$ & (0.026) & $(0.023)$ & $(0.020)$ & $(0.021)$ & (0.027) & (0.034) & $(0.022)$ & $(0.015)$ & $(0.021)$ & (0.018) \\
\hline \multirow[t]{2}{*}{$\tau_{1, R}$} & -0.133 & -0.133 & -0.134 & -0.134 & -0.089 & -0.089 & -0.090 & -0.089 & 0.004 & 0.004 & 0.004 & 0.004 & -0.107 & -0.107 & -0.108 & -0.108 \\
\hline & $(0.007)$ & $(0.007)$ & $(0.007)$ & $(0.007)$ & $(0.007)$ & $(0.007)$ & $(0.007)$ & $(0.007)$ & $(0.007)$ & $(0.007)$ & $(0.007)$ & $(0.007)$ & (0.008) & (0.008) & (0.008) & $(0.008)$ \\
\hline \multirow[t]{2}{*}{$\tau_{2, R}$} & 0.108 & 0.108 & 0.107 & 0.107 & 0.098 & 0.098 & 0.097 & 0.097 & 0.140 & 0.140 & 0.141 & 0.141 & 0.099 & 0.099 & 0.099 & 0.099 \\
\hline & $(0.005)$ & $(0.005)$ & $(0.005)$ & $(0.005)$ & $(0.005)$ & $(0.005)$ & $(0.005)$ & $(0.005)$ & $(0.006)$ & $(0.006)$ & $(0.006)$ & $(0.006)$ & $(0.005)$ & $(0.005)$ & $(0.005)$ & $(0.005)$ \\
\hline \multirow[t]{2}{*}{$\delta_{0}$} & -1.598 & -1.572 & -1.615 & -1.581 & -1.656 & -1.706 & -1.679 & -1.716 & -1.479 & -1.538 & -1.473 & -1.541 & -1.459 & -1.463 & -1.459 & -1.467 \\
\hline & $(0.042)$ & $(0.027)$ & $(0.042)$ & $(0.026)$ & $(0.045)$ & $(0.026)$ & (0.046) & $(0.026)$ & $(0.048)$ & $(0.026)$ & (0.048) & $(0.026)$ & $(0.048)$ & $(0.024)$ & $(0.049)$ & $(0.024)$ \\
\hline \multirow[t]{2}{*}{$\delta_{1}$} & 0.035 & 0.074 & 0.041 & 0.079 & -0.058 & -0.100 & -0.043 & -0.082 & -0.067 & -0.114 & -0.076 & -0.127 & -0.006 & -0.017 & -0.010 & -0.022 \\
\hline & $(0.025)$ & $(0.044)$ & $(0.025)$ & $(0.044)$ & $(0.029)$ & $(0.050)$ & $(0.030)$ & $(0.050)$ & $(0.031)$ & $(0.048)$ & $(0.031)$ & $(0.048)$ & $(0.036)$ & $(0.050)$ & $(0.036)$ & $(0.051)$ \\
\hline \multirow[t]{2}{*}{$\xi_{Q}$} & -0.021 & -0.022 & 0.005 & 0.005 & -0.296 & -0.297 & -0.187 & -0.188 & -0.813 & -0.817 & -0.811 & -0.815 & -0.768 & -0.769 & -0.834 & -0.834 \\
\hline & $(0.130)$ & $(0.205)$ & $(0.234)$ & $(0.182)$ & $(0.228)$ & $(0.204)$ & $(0.251)$ & $(0.229)$ & $(0.195)$ & $(0.198)$ & $(0.251)$ & $(0.315)$ & $(0.215)$ & $(0.152)$ & $(0.208)$ & $(0.176)$ \\
\hline \multirow[t]{2}{*}{$\varphi_{Q}$} & 0.978 & 0.978 & 0.980 & 0.980 & 0.984 & 0.984 & 0.994 & 0.994 & 0.937 & 0.936 & 0.936 & 0.936 & 0.933 & 0.933 & 0.926 & 0.926 \\
\hline & $(0.016)$ & $(0.022)$ & $(0.025)$ & $(0.020)$ & $(0.023)$ & $(0.021)$ & $(0.025)$ & $(0.023)$ & $(0.020)$ & $(0.020)$ & $(0.026)$ & $(0.032)$ & $(0.022)$ & $(0.014)$ & $(0.021)$ & $(0.018)$ \\
\hline \multirow[t]{2}{*}{$\tau_{1, Q}$} & -0.115 & -0.115 & -0.115 & -0.115 & -0.076 & -0.076 & -0.077 & -0.077 & 0.007 & 0.007 & 0.006 & 0.006 & -0.080 & -0.080 & -0.081 & -0.081 \\
\hline & $(0.010)$ & $(0.010)$ & $(0.010)$ & $(0.010)$ & $(0.008)$ & $(0.008)$ & $(0.008)$ & (0.008) & $(0.009)$ & $(0.009)$ & $(0.009)$ & $(0.009)$ & $(0.009)$ & $(0.009)$ & $(0.009)$ & $(0.009)$ \\
\hline$\tau_{2, Q}$ & 0.137 & 0.137 & 0.137 & 0.138 & 0.115 & 0.115 & 0.114 & 0.114 & 0.153 & 0.153 & 0.153 & 0.153 & 0.110 & 0.110 & 0.110 & 0.110 \\
\hline & $(0.006)$ & $(0.007)$ & $(0.007)$ & $(0.007)$ & $(0.006)$ & $(0.006)$ & $(0.006)$ & $(0.006)$ & $(0.007)$ & $(0.007)$ & $(0.007)$ & $(0.007)$ & $(0.006)$ & $(0.005)$ & $(0.006)$ & $(0.006)$ \\
\hline$\sigma_{u, Q}^{2}$ & 0.366 & 0.366 & 0.363 & 0.363 & 0.273 & 0.273 & 0.272 & 0.272 & 0.326 & 0.326 & 0.325 & 0.325 & 0.291 & 0.291 & 0.290 & 0.290 \\
\hline & $(0.008)$ & $(0.008)$ & $(0.008)$ & (0.008) & $(0.006)$ & $(0.006)$ & $(0.006)$ & $(0.006)$ & $(0.007)$ & $(0.007)$ & $(0.007)$ & $(0.007)$ & $(0.007)$ & $(0.007)$ & $(0.006)$ & $(0.006)$ \\
\hline$\rho$ & 0.911 & 0.911 & 0.911 & 0.911 & 0.927 & 0.927 & 0.927 & 0.927 & 0.932 & 0.932 & 0.932 & 0.932 & 0.946 & 0.946 & 0.946 & 0.946 \\
\hline & $(0.003)$ & $(0.003)$ & $(0.003)$ & $(0.003)$ & $(0.002)$ & $(0.002)$ & $(0.002)$ & $(0.002)$ & $(0.002)$ & $(0.002)$ & $(0.002)$ & $(0.002)$ & $(0.002)$ & $(0.002)$ & $(0.002)$ & $(0.002)$ \\
\hline$v$ & 8.935 & 8.936 & 9.146 & 9.140 & 11.886 & 11.889 & 12.130 & 12.145 & 7.139 & 7.139 & 7.295 & 7.296 & 8.808 & 8.811 & 8.850 & 8.850 \\
\hline & $(1.783)$ & $(0.814)$ & $(0.365)$ & $(0.285)$ & $(0.699)$ & $(0.279)$ & $(0.586)$ & $(0.442)$ & $(0.354)$ & $(0.385)$ & $(0.305)$ & $(0.501)$ & $(0.377)$ & $(1.920)$ & $(0.563)$ & $(0.702)$ \\
\hline$\ell_{r}$ & 13223.361 & 13223.359 & 13227.531 & 13227.518 & 14281.794 & 14281.803 & 14284.034 & 14284.063 & 13528.720 & 13528.737 & 13532.400 & 13532.409 & 14257.275 & 14257.274 & 14258.011 & 14258.012 \\
\hline $\mathscr{L}(\theta)$ & 10464.304 & 10464.722 & 10482.557 & 10482.767 & 12897.490 & 12897.626 & 12904.855 & 12905.156 & 11641.340 & 11641.923 & 11652.579 & 11653.158 & 12816.422 & 12816.466 & 12828.086 & 12828.140 \\
\hline
\end{tabular}

In-sample parameter estimates for the full sample period 2002/01/01 - 2018/04/30. Estimates refer to the TV-RGARCH (TV-RG) and ETV-RGARCH (ETV-RG) models which are based on the time-varying variance of the measurement error: $\sigma_{u_{R}, t}^{2}=\exp \left\{\delta_{0}+\delta_{1} V\left(Y_{t-1}\right)\right\}$. The function $V(\cdot)$ corresponds to the identity function for TV-(Heteroskedastic)RG and ETV-(Heteroskedastic)RG and the logarithm for TV-HRG* and ETV-HRG*. $\ell_{r}$ : partial loglikelihood. $\mathscr{L}(\theta)$ : log-likelihood. Standard errors are reported in parentheses. Parameters that are not significant at the $5 \%$ level are reported in boldface. 